\title{
On Polynomial Time Computation Over Unordered Structures
}

\author{
Andreas Blass* $\quad$ Yuri Gurevich ${ }^{\dagger} \quad$ Saharon Shelah ${ }^{\ddagger}$ \\ July 11, 2021
}

\begin{abstract}
This paper is motivated by the question whether there exists a logic capturing polynomial time computation over unordered structures. We consider several algorithmic problems near the border of the known, logically defined complexity classes contained in polynomial time. We show that fixpoint logic plus counting is stronger than might be expected, in that it can express the existence of a complete matching in a bipartite graph. We revisit the known examples that separate polynomial time from fixpoint plus counting. We show that the examples in a paper of Cai, Fürer, and Immerman, when suitably padded, are in choiceless polynomial time yet not in fixpoint plus counting. Without padding, they remain in polynomial time but appear not to be in choiceless polynomial time plus counting. Similar results hold for the multipede examples of Gurevich and Shelah, except that their final version of multipedes is, in a sense, already suitably padded. Finally, we describe another plausible candidate, involving determinants, for the task of separating polynomial time from choiceless polynomial time plus counting.
\end{abstract}

*Partially supported by NSF grant DMS-0070723 and a grant from Microsoft Research. Address: Mathematics Department, University of Michigan, Ann Arbor, MI 48109-1109, U.S.A. email: ablass@umich.edu

${ }^{\dagger}$ Microsoft Research, One Microsoft Way, Redmond, WA 98052, U.S.A. e-mail: gurevich@microsoft.com

${ }^{\ddagger}$ Partially supported by U.S.-Israel Binational Science Foundation. Address: Institute of Mathematics, Hebrew University of Jerusalem, Givat Ram, 91904 Jerusalem, Israel, and Mathematics Department, Rutgers University, New Brunswick, NJ 08903, U.S.A. e-mail: shelah@math.huji.ac.il 


\section{Introduction}

We shall be concerned with computational problems whose inputs are finite structures (for a fixed, finite vocabulary $\Upsilon$ ) and whose outputs are "yes" and "no" (or 1 and 0 , or true and false).

When $\Upsilon$ contains a binary relation symbol $\preceq$ interpreted in all input structures as a linear ordering of the underlying set, then these structures admit an easy, canonical encoding as strings. In this situation, one defines polynomial time computation on ordered structures to mean polynomial time Turing machine computation using as inputs the string encodings of the structures. Of course, polynomial time is robust, so equivalent definitions could be given using other computation models in place of Turing machines.

Turing machines that include a clock to stop the computation after a specified polynomial number of steps thus form a computation model capturing PTime on ordered structures. They constitute a logic in the broad sense defined in [11]. Immerman [14] and Vardi [17] showed that PTime on ordered structures is also captured by a logic with the look and feel traditionally associated with logics, namely fixpoint logic FP. (We shall review in Section 2 the definitions of FP and other logics mentioned in this introduction.)

For unordered input structures, the situation is quite different. One can encode such a structure as a string by first choosing a linear ordering of the underlying set. Thus, the same structure has many string encodings, and no efficient way is known to choose a preferred encoding. Following Chandra and Harel [8], one says that a problem having unordered structures as inputs is solvable in polynomial time if there is a PTime Turing machine that solves the problem when given any string encoding of the input structure (arising from any ordering of the underlying set).

This does not provide a logic in the sense of [11], because that sense requires the sentences of a logic to form a recursive set. In the case at hand, the "sentences" would be PTime Turing machines whose output is the same for any two inputs encoding the same structure. This invariance property is undecidable, so the recursivity requirement is violated.

Nor does fixpoint logic FP capture PTime on unordered structures. It cannot even express "the universe has an even number of elements" when the vocabulary $\Upsilon$ is empty.

It remains an open problem whether there is any logic at all (in the sense of [11) capturing PTime on unordered structures. It was conjectured in [11] that there is no such logic. 
There have been, however, continuing efforts to find logical systems capturing at least large parts of PTime, if not all of it. These efforts have looked primarily in two directions.'] One direction involves adding to FP additional constructs, usually in the form of quantifiers, to permit the direct expression of certain easily computable properties of unordered structures, for example the property "the universe has an even number of elements" mentioned above. The most popular of these extensions has been to add counting to the logic. There are several ways to formalize this extension; we choose the one described in [15, Ch. 4]. It involves adjoining to the input structure a second sort, consisting of the natural numbers up to the cardinality of the input set, and adding to the language terms of the form "the number of elements $x$ satisfying $\varphi(x) . "$

The second direction taken by the search for a PTime logic involves combining a standard computation mechanism with additional logical (rather than arithmetical) facilities. The relational machines of Abiteboul and Vianu 11 are of this sort, combining a Turing machine and first-order logic. Another model of this sort, more directly relevant to our purposes here, is choiceless polynomial time, $\tilde{\mathrm{CPT}}$, introduced in [4]. Here the abstract state machine model of computation [12, 2] is applied in a set-theoretic context, allowing essentially arbitrary data types over the input structure. It is shown in [5] that $\tilde{C}$ PT is strictly stronger than PTime relational machines, but even so it cannot compute the parity of an unstructured set [4]. It thus appears that this second direction produces unduly weak models. On the other hand, we shall see that $\tilde{\mathrm{CPT}}$ is capable of computing some things that are beyond the reach of $\mathrm{FP}$ plus counting.

It is therefore reasonable to combine the two directions and consider computation models (or logics) like $\tilde{\mathrm{CPT}}+$ Card, which is $\tilde{\mathrm{CPT}}$ augmented with the ability to compute cardinalities. This model was already proposed in 4 , Subsection 4.8] as worthy of further study. The present paper contains the first results of that study. The main problem, which remains open, is whether $\tilde{\mathrm{CPT}}$ plus counting captures polynomial time on unordered structures.

Most of the results we present here are concerned with specific algorithmic problems that are solvable in PTime but appear to be at the borderline of expressibility in logics like $\tilde{C}$ PT plus counting. Several of them are plausible candidates for separating PTime from $\tilde{\mathrm{CPT}}$ plus counting.

\footnotetext{
${ }^{1} \mathrm{~A}$ third direction, studied by Gire and Hoang [9], involves a form of restricted nondeterminism. This direction looks promising, but we do not address it in this paper.
} 
We begin with work motivated by the result from [4] that bipartite matching is not in $\tilde{\mathrm{CPT}}$. The proof of this involved exceptionally simple instances of the bipartite matching problem. In the traditional picture of bipartite matching, where the input consists of a set of boys, a set of girls, and a (symmetric) "willing to marry" relation between them, the instances used in [1] can be described as follows. First suppose there are $2 n$ boys and $2 n$ girls, divided into two gangs of $n$ boys and $n$ girls each; a boy and a girl are willing to marry if and only if they belong to the same gang. Obviously, a complete matching exists in this case. Next suppose one of the boys defects from his gang and joins the other, while all girls remain in their original gangs. Obviously there is no matching now. But a $\tilde{\mathrm{CPT}}$ program cannot distinguish these two situations if $n$ is sufficiently large compared to the program (件, Thm. 43]). This specific deficiency can evidently be removed by adding to $\tilde{\mathrm{CPT}}$ the ability to count, but it seems that this success depends on the very simple structure of the "willing to marry" relation. There seems to be no way to extend this result to general instances of bipartite matching. Thus, there was some hope that bipartite matching would serve to separate PTime from $\tilde{C}$ PT + Card. That hope is dashed here in Section 3, where we present a $\tilde{C P T}+$ Card algorithm to decide whether a bipartite graph has a complete matching. In fact, we show the rather surprising result that the existence of a complete matching can be expressed in FP+Card.

In an effort to separate PTime from $\tilde{\mathrm{CPT}}+\mathrm{Card}$, we next turn to the two known types of examples separating PTime from FP+Card. These examples involve certain graphs defined by Cai, Fürer, and Immerman [7] and structures called multipedes introduced by two of the present authors [13]. For the reader's convenience, we recapitulate the relevant information from [7] and 13. Then we discuss how the constructions from these papers lead naturally to queries that are in PTime but not in FP+Card. We show that, for suitably padded versions of the examples from [7] and also for the so-called 4-multipedes of 13 (without padding), these queries are in CPT even without counting. Thus, these examples show that FP+Card does not include $\tilde{\mathrm{CPT}}$ and is strictly included in $\tilde{\mathrm{CPT}}+$ Card.

There are very similar queries, using the graphs from [7] without padding or using the 3-multipedes from [13], which are still in PTime (by somewhat trickier proofs than the versions in the preceding paragraph) but which we do not see how to express in $\tilde{C} P T+$ Card. So perhaps one of these will give the desired separation.

Finally, motivated by the use of linear algebra modulo 2 in some of the 
preceding arguments, we consider the computation of (suitably presented) determinants. We show that the question whether a matrix over a finite field or over the integers is singular (i.e., has zero determinant) is in FP+Card. It is not in $\tilde{\mathrm{CPT}}$, even over the two-element field; the proof of this uses the zeroone law proved by one of us in [16] and discussed by the other two in [3]. The computation of determinants (in contrast to merely deciding whether they are zero) over the prime field $\mathbb{Z} / p$ for an odd prime $p$ is in PTime, but we do not know whether it is in $\tilde{\mathrm{CPT}}+$ Card.

\section{Background}

In this section we review the logics and the computation models relevant to this paper, namely

- fixpoint logic (FP),

- finite variable infinitary logic $\left(L_{\infty, \omega}^{\omega}\right)$,

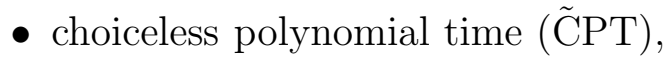

and their extensions by counting, $\mathrm{FP}+\mathrm{Card}, C_{\infty, \omega}^{\omega}$, and $\tilde{\mathrm{CPT}}+\mathrm{Card}$, respectively. (The notation "+Card" stands for adding cardinality to the logic.) We refer the reader to [15] for details about FP, FP+Card, $L_{\infty, \omega}^{\omega}$, and $C_{\infty, \omega}^{\omega}$ and to [4] for details about $\tilde{\mathrm{CPT}}$ and $\tilde{\mathrm{CPT}}+$ Card.

Fixpoint logic FP is obtained by adding to ordinary first-order logic the (inflationary) fixpoint operator defined as follows. If $X$ is an $r$-ary relation symbol not in $\Upsilon$, if $\varphi(X, \vec{x})$ is a formula of the vocabulary $\Upsilon \cup\{X\}$, and if $\vec{x}$ is an $r$-tuple of distinct variables, then $\mathrm{FP}_{X, \vec{x}} \varphi$ is used as an $r$-ary relation symbol. It is interpreted as the fixed point obtained by starting with the empty relation and iterating the operation

$$
R \mapsto R \cup\{\vec{x}: \varphi(R, \vec{x})\}
$$

(For a precise formulation, one should fix an $\Upsilon$-structure and values for all free variables of $\varphi$ except $\vec{x}$, and then the operation above should be interpreted using these data; see [15].)

Fixpoint logic with counting, called $\mathrm{FP}+\mathrm{C}$ in 15 but $\mathrm{FP}+\mathrm{Card}$ here (to conform with the notation $\tilde{\mathrm{CPT}}+\mathrm{Card}$ ), is obtained from FP by the following modifications. First, every input structure $\mathfrak{A}$, with underlying set 
$A$, is replaced by a two-sorted structure $\mathfrak{A}^{*}$ in which one sort is $\mathfrak{A}$ and the other is the initial segment $\{0,1, \ldots,|A|\}$ of the natural numbers with the successor function. Second, for each variable $x$ and formula $\varphi(x)$, there is a term $(\sharp x) \varphi(x)$ denoting the number (an element of the new sort) of values of $x$ that satisfy $\varphi(x)$. Fixpoint operations are allowed to define relations on either or both sorts; in particular, addition and multiplication are definable on the number sort insofar as their values don't overflow the available range of numbers.

The infinitary logic $L_{\infty, \omega}^{\omega}$ is obtained from ordinary first-order logic by making two changes. First, allow conjunctions and disjunctions of arbitrary, possibly infinite sets of formulas. (The logic resulting from this first change is called $L_{\infty, \omega}$.) Second, require each formula to use only a finite number of variables, where both free and bound variables are counted but the same variable may be re-used, i.e., it may occur both free and bound and possibly bound many times. $L_{\infty, \omega}^{k}$ is the sub-logic in which the number of variables in a formula is required to be at most the natural number $k$. It is known (see for example [15, Cor. 1.30]) that FP is included in $L_{\infty, \omega}^{\omega}$ in the sense that, for every formula of FP, there is a formula of $L_{\infty, \omega}^{\omega}$ that is semantically equivalent, i.e., satisfied by the same tuples of elements in the same structures.

The logic $L_{\infty, \omega}^{\omega}$ could be extended by counting terms just as FP was extended to FP+Card, but we shall instead follow [15] and use the more traditional counting quantifiers. The logic $C_{\infty, \omega}^{\omega}$ is obtained from $L_{\infty, \omega}^{\omega}$ by adding the quantifiers $\exists^{\geq m}$ for all natural numbers $m$, semantically interpreted as "for at least $m$ values of". It is shown in [15, Cor. 4.20] that $C_{\infty, \omega}^{\omega}$ includes FP+Card.

Since $\tilde{\mathrm{CPT}}$ is newer and less widely known than the fixpoint and infinitary logics discussed above, we describe it in somewhat more detail, but for a full definition we refer to [1]. $\tilde{\mathrm{CPT}}$ is the polynomial time fragment of a programming language, BGS, defined as follows. Inputs to a computation are finite structures for a vocabulary $\Upsilon$; each program is associated with a fixed $\Upsilon$, but different programs can use different $\Upsilon$ 's and thus admit different sorts of inputs. A computation proceeds in discrete stages, the state at any moment being a structure of the following sort. Its underlying set $\mathrm{HF}(I)$ consists of the underlying set $I$ of the input structure (regarded as a set of atoms, i.e., non-sets) plus all hereditarily finite sets over $I$, that is, all subsets of $I$, all sets whose members are either such subsets or members of $I$, etc. In other words, $\mathrm{HF}(I)$ is the smallest set having among its members all its finite subsets and all the members of $I$. Notice that $\operatorname{HF}(I)$ contains the natural 
numbers, coded as von Neumann ordinals,

$$
0=\varnothing, \quad 1=\{\varnothing\}, \quad \ldots, \quad n=\{0,1, \ldots, n-1\}, \quad \ldots
$$

For computational purposes, this representation of the natural numbers is equivalent (in the BGS context) to unary notation for the natural numbers. So we assume from now on that natural numbers (and in fact all integers) are available, in unary notation, along with the basic arithmetical operations. In Section 6, we shall also need binary notations; details about that representation will be given there.

We use 0 and 1 to represent the truth values false and true, respectively. Thus predicates can be regarded as $\{0,1\}$-valued functions. The structure giving a state of the computation has the following basic functions:

- the functions and relations of the input structure, relations being regarded as $\{0,1\}$-valued functions, and all functions being extended to have value 0 when any input is not in $I$,

- the logical functions: $=$, true, false, $\neg, \wedge, \vee$,

- the set-theoretic functions $\in, \varnothing$, Atoms, $\bigcup$, TheUnique, Pair,

- finitely many dynamic functions, including Halt and Output.

Here Atoms means the set $I$ of atoms (as opposed to sets) in $\mathrm{HF}(I)$. The function $U$ sends any $x$ to the union of all the sets that are members of $x$, TheUnique $(x)$ is the unique member of $x$ if $x$ is a set having exactly one member (and 0 otherwise), and $\operatorname{Pair}(x, y)$ is the set $\{x, y\}$. The dynamic functions are constant with value 0 in the computation's initial state but acquire more interesting values as the computation proceeds. The vocabulary of a BGS program has symbols for all these functions. The symbols for the input relations (as opposed to functions), the logic symbols, $\in$, the dynamic functions Halt and Output, and possibly some other dynamic function symbols are called Boolean; their values are always 0 or 1. (If we were interested in computing results other than "yes" and "no", then we would not declare Output to be Boolean.)

The meaningful expressions of the programming language BGS are terms and rules. Terms are built from the function symbols described above and variables in the usual way, with the addition of the term-forming construction

$$
\{t(v): v \in r: \varphi(v)\}
$$


where $t$ and $r$ are terms, $\varphi$ is a Boolean term (i.e., one whose outermost constructor is a Boolean function symbol), and $v$ is a variable not free in $r$. (By writing $v$ in the contexts $t(v)$ and $\varphi(v)$, we mean to indicate only that $v$ is allowed to occur free there, not that it must occur free, nor that other variables cannot occur free.) The interpretation of the term $\{t(v): v \in r$ : $\varphi(v)\}$ is the set of values of $t(v)$ for all values of $v$ that are members of the value of $r$ and make $\varphi$ true. When $\varphi$ is true, we sometimes omit it from the notation and write simply $\{t(v): v \in r\}$.

We note for future use that there are terms representing the union of two sets,

$$
a \cup b=\bigcup \operatorname{Pair}(a, b)
$$

and the traditional set-theoretic coding of ordered pairs

$$
\langle a, b\rangle=\{\{a\},\{a, b\}\}=\operatorname{Pair}(\operatorname{Pair}(a, a), \operatorname{Pair}(a, b)) .
$$

Rules are built by the following inductive construction. Each rule defines, in an obvious way, a set of updates of the state, provided values are specified for the rule's free variables.

- Skip is a rule (producing no updates).

- If $f$ is a dynamic function symbol, say $j$-ary, and $t_{0}, t_{1}, \ldots, t_{j}$ are terms, with $t_{0}$ Boolean if $f$ is, then

$$
f\left(t_{1}, \ldots, t_{j}\right):=t_{0}
$$

is a rule.

- If $R_{0}$ and $R_{1}$ are rules and $\varphi$ is a Boolean term, then

$$
\text { if } \varphi \text { then } R_{0} \text { else } R_{1} \text { endif }
$$

is a rule.

- If $R_{0}(v)$ is a rule, $v$ is a variable, and $r$ is a term in which $v$ is not free, then

$$
\text { do forall } v \in r, R_{0}(v) \text { enddo }
$$

is a rule. 
The notion of free variable, used in these definitions, is defined in the usual way, with the term constructor $\{t(v): v \in r: \varphi(v)\}$ and the rule constructor do forall $v \in r, R_{0}(v)$ enddo binding the variable $v$.

A program is a rule with no free variables. To fire a program in a state is to modify the dynamic functions of the state according to all the updates produced by the program except that, if two of these updates are contradictory (i.e., update the same dynamic function at the same tuple of arguments to different values), then none of the updates are executed. A run of a program on an input is a sequence of states in which the first state is the initial state determined by the input structure (as above, with all dynamic functions constantly 0) and each subsequent state is obtained from its predecessor by firing the program. The result of the computation is the value of Output at the first stage where Halt has the value true (i.e., 1). (It would do no harm to automatically stop all runs whenever Halt has the value true or to insist that programs produce no updates in this situation.) If Halt never becomes true then the computation fails to produce an output.

A PTime bounded BGS program is a BGS program $\Pi$ together with two polynomials $p(n)$ and $q(n)$. A run of $(\Pi, p(n), q(n))$ on input $I$ is a run of $\Pi$ consisting of at most $p(|I|)$ stages and having at most $q(|I|)$ active elements. We do not reproduce here the definition of "active" from [4] but remark that, roughly speaking, an element of $\operatorname{HF}(I)$ is active if it is either involved in an update during the run or a member of something involved in an update, or a member of a member, etc.

For the purposes of this paper, we define $\tilde{\mathrm{CPT}}$ as the class of Boolean queries decidable by PTime bounded BGS programs. (A broader definition, using a three-valued logic to accommodate computations where Halt never becomes true, was used in 四, but we will not need to use it here.)

We observe that $\tilde{\mathrm{CPT}}$ includes the expressive power of first order logic. The propositional connectives were included among the basic functions on $\mathrm{HF}(I)$, and the quantifiers over the input structure can be simulated because $(\exists v \in I) \varphi(v)$ is equivalent to

$$
0 \in\{0: v \in \text { Atoms }: \varphi(v)\} .
$$

Furthermore, CिP includes the expressive power of fixpoint logic, for the iteration defining a fixpoint can be simulated by the iteration involved in the notion of run. In fact, it was shown in $₫$, Thm. 20] that $\tilde{C}$ PT can simulate the PTime relational machines of Abiteboul and Vianu [1]; it is known that these can compute all FP-definable queries. 
We shall need several times the observation that C̃PT includes all PTime (and in fact exponential time) computations on sufficiently small parts of the input structure. Specifically, if the input structure has a definable subset $P$ with $|P| ! \leq|I|$, then a BGS program can first produce, in a parallel computation, $|P|$ ! subprocesses each of which knows a linear ordering of $P$. Then each of these subprocesses can run a PTime algorithm on its ordered version of $P$. If the PTime algorithm produces the same answer for all orderings, then these subprocesses will all give Output that value, so the overall algorithm produces this answer. And the inequality $|P| ! \leq|I|$ implies that this is a PTime bounded BGS program, so the result of the computation is in $\tilde{C P T}$. For the details of this argument, see the proof of 4 , Thm. 21]. Similarly, under the weaker assumption that $2^{|P|} \leq|I|$, a BGS program can produce, in polynomial time, all the subsets of $P$.

To add counting to $\tilde{C}$ PT, we simply include, in every state, the additional function Card that sends every set to its cardinality (considered as a von Neumann ordinal) and sends atoms to 0 . The resulting complexity class is called $\tilde{\mathrm{CPT}}+$ Card.

The cardinality function makes it possible to carry out, in a single step, the operations of addition and multiplication on von Neumann ordinals. Indeed, we can express $a+b$ as the cardinality of

$$
a \cup\{\langle 0, x\rangle: x \in b\},
$$

and $a b$ is the cardinality of the cartesian product

$$
a \times b=\bigcup\{\{\langle x, y\rangle: x \in a\}: y \in b\} .
$$

Remark 1 Theorem 8 of [1] says, roughly speaking, that every object activated during a run of a PTime BGS program was "looked at" during that run. This is no longer true when we add Card to the computation model. The ordinal Card $(x)$ can be active in a state without all its predecessors being looked at. For example, if the input is a linearly ordered set of size $n$, a computation can, since addition is available, initialize a nullary dynamic $c$ to 1 and then perform $n$ steps doubling $c$ at each step. Then $2^{n}$ is active in the final state, but most of the ordinals below it have not been looked at.

Intuitively, the computation just described should not count as polynomial time, for it involves parallel processes indexed by sets of size exponentially big compared to the input. Our definition of PTime in the BGS 
context agrees with this intuition, for the number of active elements in this computation is exponential. (The number of critical elements, in the sense of [4] is only polynomial, so it is important to include members of critical elements, their members, etc. in the definition of active elements and thus in the definition of PTime.

Remark 2 BGS was designed for theoretical purposes. Some of its conventions were designed to simplify analysis of programs and thus are unnatural from a programming point of view. In this paper, we retain those conventions and work around them where necessary. But for actual programming, these conventions should be modified. In particular, arithmetic should be available directly rather than being coded in the von Neumann ordinals. The input itself should in general be a metafinite structure in the sense of [10].

The following diagram indicates the relationships between the various logics and complexity classes considered here. Arrows represent inclusion relationships.

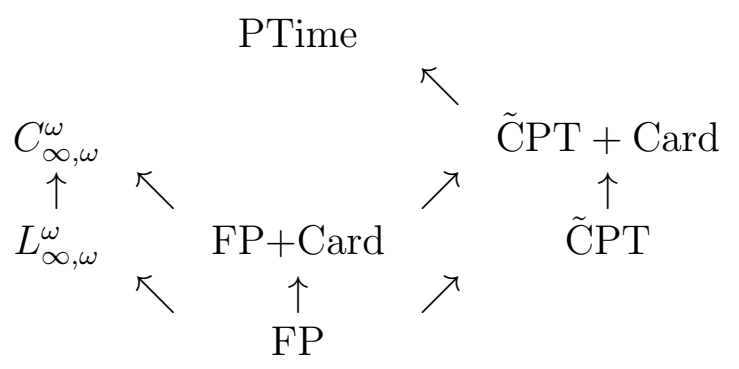

In the following sections, we consider various specific problems and try to determine which of these logics and complexity classes contain them.

\section{Bipartite Matching}

\subsection{Statement of the Problem}

Definition 3 A bipartite graph consists of two finite sets $A$ and $B$ with an adjacency relation $R \subseteq A \times B$.

We denote a bipartite graph by $\langle A, B, R\rangle$. It makes no difference whether we regard it as a two-sorted structure with one binary predicate $R$ or as a 
one-sorted structure with, in addition to $R$, unary predicates for $A$ and $B$. Even if we adopt the two-sorted viewpoint, we assume whenever convenient that $A$ and $B$ are disjoint.

Definition 4 A matching in a bipartite graph $\langle A, B, R\rangle$ is a partial one-toone function $M$ from $A$ into $B$ which, considered as a binary relation (a set of ordered pairs), is a subset of $R$. We call

a matching complete if its domain is all of $A$.

Definition 5 Bipartite matching is the following decision problem. The input is a bipartite graph $\langle A, B, R\rangle$ and the question is whether it has a complete matching.

In these definitions, we did not require $|A|=|B|$, so a complete matching may have its range strictly included in $B$. Everything we say about the bipartite matching problem remains true if we restrict the inputs to be bipartite graphs $\langle A, B, R\rangle$ with $|A|=|B|$.

It was shown in [4] that the bipartite matching problem is not in $\tilde{\mathrm{CPT}}$. The proof of this fact exploited the inability of $\tilde{\mathrm{CPT}}$ to count. For the particular graphs used in that proof, the decision problem would become easy if counting were available, but this observation does not apply to more general instances of bipartite matching. The question thus arises whether the bipartite matching problem is in $\tilde{\mathrm{CPT}}+$ Card.

We present here a somewhat surprising affirmative answer. In fact, we show that bipartite matching is expressible in FP+Card.

\subsection{Known Algorithms}

In this subsection we describe two well-known approaches to the bipartite matching problem. Neither provides a solution in $\tilde{\mathrm{CPT}}+$ Card, but both will play a role in the solution.

The first is an algorithm which we call the path algorithm. It works with (incomplete) matchings, starting with the empty one and at each stage either replacing the current matching by a larger one or determining that no complete matching can exist.

To describe a step of this algorithm, let $M$ be the current matching. If it is complete, then output "yes" and halt. If it is incomplete, then proceed as 
follows. Consider the directed graph whose vertex set is $A \dot{\cup} B$ (we invoke our standing assumption that $A$ and $B$ are disjoint whenever convenient) and whose directed edges are

- all $(a, b) \in R-M$ and

- the converses $(b, a)$ of all $(a, b) \in M$.

In other words, start by regarding all pairs $(a, b) \in R$ as directed edges from $a$ to $b$, but then reverse the direction of those pairs that are in the current matching $M$.

If this directed graph has a directed path from a vertex $a \in A-\operatorname{Dom}(M)$ to a vertex $b \in B-\operatorname{Range}(M)$, then choose one such path, and let $P$ be the corresponding set of pairs in $R$ (i.e., take the edges in the path and reverse the direction of those that are in $M$, so as to get pairs in $R$ ). Notice that, except for the endpoints $a$ and $b$, every vertex in our path has two incident edges in $P$, one of which is in $M$ and the other not in $M$; the endpoints, of course, have only one incident edge (each) and it is not in $M$. This implies immediately that the symmetric difference $M \triangle P$ is a matching of cardinality one greater than that of $M$. Proceed to the next step with $M \triangle P$ as the current matching.

If the directed graph has no path from a vertex $a \in A-\operatorname{Dom}(M)$ to a vertex $b \in B$ - Range $(M)$, then output "no" and halt.

This completes the description of the algorithm, but it should be accompanied by an explanation of why the "no" answer in the last situation is correct. (All other aspects of correctness - eventual termination and correctness of the "yes" answers - are obvious.) So suppose that, at some step of the algorithm, there is no directed path of the required sort. Since the algorithm has not yet halted with "yes", there are points $a \in A-\operatorname{Dom}(M)$; fix one such $a$. Let $X$ and $Y$ be the sets of all vertices in $A$ and $B$, respectively, that are reachable from $a$ by directed paths in the digraph under consideration. By assumption, $Y \subseteq$ Range $(M)$. Furthermore, by the definition of the digraph, $X$ contains all the points that are matched by $M$ with points in $Y$. In addition, $X$ contains the point $a$, which isn't matched with anything. Therefore, $|X|>|Y|$. It is easy to check, using again the definition of the digraph, that every pair in $R$ whose first component is in $X$ has its second component in $Y$. Thus, if there were a complete matching for $\langle A, B, R\rangle$, it would have to map $X$ one-to-one into $Y$, which is impossible as $|X|>|Y|$. 
Therefore, no such matching can exist, and the algorithm's "no" answer is correct.

The preceding discussion not only establishes the correctness of the path algorithm but also essentially proves the celebrated "marriage theorem", often called Hall's theorem. For the history of this theorem see [6, page 54].

Theorem 6 ("Marriage") A bipartite graph $\langle A, B, R\rangle$ admits a complete matching if and only if, for every $X \subseteq A$, its set of $R$-neighbors

$$
Y=\{b \in B:(\exists x \in X)(x, b) \in R\}
$$

has cardinality $|Y| \geq|X|$.

Proof As mentioned above, a complete matching must map every $X$ oneto-one into the corresponding $Y$, so $|Y| \geq|X|$. This proves the "only if" part of the theorem.

For the "if" part, suppose $\langle A, B, R\rangle$ has no complete matching. Then the path algorithm must eventually output "no," and when it does it has found, according to the discussion above, an $X$ (namely the set of points reachable in the digraph from an $a \in A-\operatorname{Dom}(M))$ for which the corresponding $Y$ has $|Y|<|X|$.

A second approach to the bipartite matching problem would be to check the condition in the marriage theorem. This second approach is clearly not in polynomial time, for there are exponentially many $X$ 's to check. It is choiceless, as the computations for all $X$ 's can be done in parallel (after all the $X$ 's have been generated, in another parallel computation), but, as pointed out in [4], choicelessness is hardly relevant in the absence of a bound on the number of activated sets. (There is a little bit of relevance, since to simulate choice by parallel computation one generally needs to activate $n$ ! sets, where $n$ is the input size, and the algorithm based on the marriage theorem activates only approximately $2^{n}$ sets.)

The path algorithm, by contrast, clearly runs in polynomial time, since it will terminate after at most $n$ steps and each step consists mainly of testing the existence of paths between certain vertices, which can be done in polynomial time. Unfortunately, this algorithm requires arbitrary choices. Whether the required path exists at any step can be decided choicelessly (see ઔ, Section 1]), but the algorithm requires choosing one such path in order to form the matching $M \triangle P$ for the next step to use. 
In fact, since the path algorithm not only decides whether a complete matching exists but, when the answer is "yes," produces one, it clearly cannot be choiceless, for some bipartite graphs have complete matchings but none that are invariant under all the graph's automorphisms. For example, consider the case where $|A|=|B| \geq 2$ and $R=A \times B$.

We record for future reference that, if the input is given with a linear order (i.e., if each of $A$ and $B$ is linearly ordered), then no further choices are needed by the path algorithm. When it searches for a path, it can do a depth-first search, going through vertices in the given order, and use the first path that it finds.

\subsection{A Choiceless Polynomial Time Algorithm}

In this subsection, we describe a $\tilde{\mathrm{CPT}}+$ Card algorithm that solves the bipartite matching problem. The description will be informal, but it should be clear that the algorithm could be programmed in BGS augmented by the cardinality function Card and that it would run in polynomial time. Thus, it shows that bipartite matching is in $\tilde{\mathrm{CPT}}+$ Card. Later, we shall prove, somewhat more formally, that the algorithm enables us to express "there is a complete matching" in the language FP+Card; this implies formally that the problem is in $\tilde{\mathrm{CPT}}+$ Card.

The algorithm proceeds in three phases, given a bipartite graph $\langle A, B, R\rangle$ as input.

In phase 1 , we partition $A$ and $B$ into subsets $A_{i}(i \in I)$ and $B_{j}(j \in J)$ respectively in such a way that

- for each $i$ and each $j$, all the vertices in $A_{i}$ have the same number of $R$-edges to $B_{j}$. That is,

$$
\left(\forall a, a^{\prime} \in A_{i}\right) \quad\left|\left\{b \in B_{j}:(a, b) \in R\right\}\right|=\left|\left\{b \in B_{j}:\left(a^{\prime}, b\right) \in R\right\}\right|,
$$

- symmetrically,

$$
\left(\forall b, b^{\prime} \in B_{j}\right) \quad\left|\left\{a \in A_{i}:(a, b) \in R\right\}\right|=\left|\left\{a \in A_{i}:\left(a, b^{\prime}\right) \in R\right\}\right|,
$$

and

- the index sets $I$ and $J$ have canonical linear orderings. 
This is achieved by the following procedure, called the "stable coloring algorithm" in 15.

We proceed in steps, having at each step a partition of $A$ and a partition of $B$, together with, for each of these partitions, a linear ordering of the blocks. As long as the current partitions are not of the desired sort, they will be refined, i.e., replaced with new partitions each block of which is included in a block of the corresponding old partition. We begin with each of $A$ and $B$ trivially partitioned into a single piece (so there is no question about the linear ordering of blocks).

Suppose, at some stage, we have a partition that does not satisfy the requirements listed above. Since we have linear orderings as in the third requirement, there must be a violation of one or both of the first two requirements.

Replace each block $A_{i}$ by a sequence of subblocks determined as follows. To each $a \in A_{i}$ assign a vector consisting of the cardinalities $\mid\left\{b \in B_{j}\right.$ : $(a, b) \in R\} \mid$ listed in order of the blocks $B_{j}$. Each vector so obtained will give one subblock, consisting of all the $a \in A_{i}$ that produced that vector. The subblocks within $A_{i}$ are ordered according to the lexicographic ordering of their vectors. Subblocks coming from different blocks $A_{i}$ and $A_{i^{\prime}}$ are ordered as those blocks were ordered in the given partition.

Replace each block $B_{j}$ by subblocks and linearly order these subblocks analogously.

Since at least one of the first two requirements was violated, at least one of our two partitions will be properly refined. Thus, the number of steps of this sort is bounded by (slightly less than) the number of vertices in $A \cup \dot{\cup} B$. Therefore, phase 1 must terminate, and when it does it has provided partitions satisfying all our requirements.

In phase 2 we first replace $R$ by the following (possibly) larger relation:

$$
R^{+}=\bigcup\left\{A_{i} \times B_{j}:\left(A_{i} \times B_{j}\right) \cap R \neq \varnothing\right\} .
$$

In other words, as soon as one vertex in $A_{i}$ is $R$-joined to one vertex in $B_{j}$ (and therefore, by the requirements on the partition, every vertex in $A_{i}$ is joined to at least one vertex in $B_{j}$, and vice versa), $R^{+}$joins every vertex in $A_{i}$ to every vertex in $B_{j}$.

Then, using the linear ordering of the blocks produced in phase 1, we create an isomorphic copy $\left\langle A^{\prime}, B^{\prime}, R^{\prime}\right\rangle$ of $\left\langle A, B, R^{+}\right\rangle$in which the vertex sets $A^{\prime}$ and $B^{\prime}$ are equipped with canonical linear orderings. To do this, let $A^{\prime}$ 
consist of triples $(0, i, r)$ where $i \in I$ and $r$ is a natural number in the range $0 \leq r<\left|A_{i}\right|$. The idea is that the $\left|A_{i}\right|$ triples whose second component is $i$ act as a substitute for the members of $A_{i}$. Define $B^{\prime}$ analogously, using triples $(1, j, s)$, and let

$$
\begin{aligned}
((0, i, r),(1, j, s)) \in R^{\prime} & \Longleftrightarrow(a, b) \in R^{+} \text {for some }(\text { all }) a \in A_{i}, b \in B_{j} \\
& \Longleftrightarrow(a, b) \in R \text { for some } a \in A_{i}, b \in B_{j} .
\end{aligned}
$$

Notice that $\left\langle A^{\prime}, B^{\prime}, R^{\prime}\right\rangle$ is isomorphic to $\left\langle A, B, R^{+}\right\rangle$, but there is no canonical choice of an isomorphism. To choose a specific isomorphism we would need to choose a linear ordering of each of the sets $A_{i}$ and $B_{j}$. Fortunately, the algorithm doesn't need any isomorphism, so it remains choiceless.

Finally, in phase 3, we apply the path algorithm to determine whether $\left\langle A^{\prime}, B^{\prime}, R^{\prime}\right\rangle$ has a complete matching. No arbitrary choices are involved here, since $A^{\prime}$ and $B^{\prime}$ are (unlike $A$ and $B$ ) canonically linearly ordered: Their elements are triples whose second components come from the index sets $I$ and $J$, for which phase 1 provided a linear order, and whose first and third components are natural numbers. So we can use the lexicographic order on the triples.

Output "yes" or "no" according to whether $\left\langle A^{\prime}, B^{\prime}, R^{\prime}\right\rangle$ has a complete matching or not.

This completes the description of the algorithm. It should be clear that it is in $\tilde{\mathrm{CPT}}+$ Card, but there is a real question about its correctness. The next subsection addresses that question.

\subsection{Correctness Proof}

If the algorithm presented in the last subsection outputs "no," this means that there is no complete matching in $\left\langle A^{\prime}, B^{\prime}, R^{\prime}\right\rangle$, hence no complete matching in the isomorphic graph $\left\langle A, B, R^{+}\right\rangle$, and hence no complete matching in the original graph $\langle A, B, R\rangle$, because $R \subseteq R^{+}$so any complete matching for $R$ would also be one for $R^{+}$.

If, on the other hand, the algorithm ouputs "yes," then there is a complete matching for $\left\langle A, B, R^{+}\right\rangle$, but this need not be a complete matching for $\langle A, B, R\rangle$, since it could use edges from $R^{+}-R$. Thus, the following lemma is needed to establish the correctness of the algorithm.

Lemma 7 In the situation of the preceding subsection, if $\left\langle A, B, R^{+}\right\rangle$has a complete matching, then so does $\langle A, B, R\rangle$. 
Proof Fix a complete matching $M$ for $\left\langle A, B, R^{+}\right\rangle$.

We first define a (reasonably fair) allocation of responsibility, among the edges $(a, b) \in R$, for the edges $(p, q) \in R^{+}$. By definition of $R^{+}$, the fact that it contains $(p, q)$ is caused by the presence in $R$ of some edges $(a, b)$ between the same blocks $A_{i}$ and $B_{j}$. If the number of such edges in $R$ is $n_{i j}$, then we allocate responsibility for $(p, q)$ equally among them, assigning each such $(a, b)$ the amount $1 / n_{i j}$ of responsibility. Thus, the total responsibility (of all $(a, b))$ for one edge $(p, q)$ is 1 , and the responsibility is shared by the $R$-edges between the same blocks as $(p, q)$.

If $(p, q)$ is an edge in $R^{+}$between blocks $A_{i}$ and $B_{j}$ and $(a, b)$ is an edge in $R$ but not between blocks $A_{i}$ and $B_{j}$ then the responsibility of $(a, b)$ for $(p, q)$ is zero. If $S$ is a subset of $R$ then the responsibility of $S$ for an edge $(p, q) \in R^{+}$is the sum of the responsibilities of the edges in $S$ for $(p, q)$. The responsibility of $S$ for a subset of $R^{+}$is the sum of the responsibilities of $S$ for the edges in the subset. Further, a vertex $v$ gives rise to a subset $S(v)$ of $R$, namely the set of edges of $R$ incident to that vertex. A set $V$ of vertices also gives rise to a subset of $R$, namely the union of the sets $S(v)$ where $v$ ranges over $V$. The allows us to speak about the responsibility of a vertex or a set of vertices for an edge or a set of edges in $R^{+}$.

Because all vertices in $A_{i}$ have the same number of $R$-edges to $B_{j}$, they all have equal responsibility for any $(p, q)$ joining these blocks in $R^{+}$, namely responsibility $1 /\left|A_{i}\right|$. (In more detail: Each of these vertices is incident to $n_{i j} /\left|A_{i}\right|$ edges to $B_{j}$, and each of these edges bears responsibility $1 / n_{i j}$ for $(p, q)$. So each vertex has responsibility $1 /\left|A_{i}\right|$ for $(p, q)$.)

Let $X$ be an arbitrary subset of $A$, and let $Y$ be, as in the marriage theorem, the set of all vertices in $B$ that have an edge in $R$ from some vertex in $X$. We shall prove that $|X| \leq|Y|$; then the marriage theorem will provide the required matching for $\langle A, B, R\rangle$.

Temporarily restrict attention to one block $A_{i}$. We consider the total responsibility of vertices in $X \cap A_{i}$ for the edges of $M$ (the fixed matching for $R^{+}$) that connect $A_{i}$ with $B$. As noted above, each vertex in $A_{i}$ has the same responsibility $1 /\left|A_{i}\right|$ for each such edge, so the vertices in $X \cap A_{i}$ have proportionate responsibility $\left|X \cap A_{i}\right| /\left|A_{i}\right|$ for each such edge of $M$. There are, since $M$ is a complete matching, exactly $\left|A_{i}\right|$ such edges. Therefore, the total responsibility of all vertices in $X \cap A_{i}$ for edges in $M$ (from $A_{i}$ to $B$ ) is $\left|X \cap A_{i}\right|$. We wrote "from $A_{i}$ to $B$ " in parentheses, because it can safely be omitted; vertices in $A_{i}$ have, by definition, no responsibility for edges (of $M$ or otherwise) originating in other blocks $A_{i^{\prime}}$. 
Now consider all the blocks, and sum over $i$ the result of the preceding paragraph. The total responsibility of all vertices in $X$ for all edges in $M$ is exactly $|X|$. Recalling how responsibility of vertices was defined, we can restate the result as: The total responsibility, for edges in $M$, of all $R$-edges originating in $X$ is exactly $|X|$.

Now we repeat, as far as possible, the preceding three paragraphs "from the other side," i.e., starting with a fixed block $B_{j}$ in $B$ and computing the total responsibility of the vertices in $Y \cap B_{j}$ for the edges in $M$ (from $A$ to $B_{j}$ ). It is their proportionate share, $\left|Y \cap B_{j}\right| /\left|B_{j}\right|$, of the total responsibility of $B_{j}$ for the $M$-edges that end in $B_{j}$. So far, this is exactly analogous to the preceding argument, but the next step is slightly different. Although the domain of $M$ is, by completeness, all of $A$, its range need not be all of $B$. Thus, the number of $M$-edges ending in $B_{j}$ is $\leq\left|B_{j}\right|$ (as $M$ is one-to-one), but equality need not hold. Therefore, we can conclude only that the total responsibility of $Y \cap B_{j}$ for $M$-edges is $\leq\left|Y \cap B_{j}\right|$.

Summing over all blocks $B_{j}$, we find that the total responsibility for $M$ edges of all vertices in $Y$ is $\leq|Y|$. As before, we rephrase this in terms of responsibility of edges: The total responsibility, for edges in $M$, of all $R$-edges ending in $Y$ is $\leq|Y|$.

Finally, we recall that, by definition of $Y$, every $R$-edge originating in $X$ must end in $Y$. Therefore

$$
\begin{aligned}
|X|= & \text { total responsibility for } M \text {-edges of } \\
& \text { edges originating in } X \\
\leq & \text { total responsibility for } M \text {-edges of } \\
& \text { edges ending in } Y \\
\leq & |Y| .
\end{aligned}
$$

This completes the verification that $\langle A, B, R\rangle$ satisfies the condition in the marriage theorem and therefore has a perfect matching.

\subsection{Fixed-Point Logic With Counting}

In this subsection we indicate how to express the existence of a complete matching in the extension FP+Card of first-order logic by fixed-point operators and counting.

First, observe that what Otto calls the stable coloring in [15, Section 2.2] amounts to the partitions $\left\{A_{i}: i \in I\right\}$ and $\left\{B_{j}: j \in J\right\}$ produced by our 
algorithm together with the linear ordering of index sets construed as a preordering of $A$ and $B$. The pre-ordering has $x \prec y$ if and only if $x$ is in an earlier block than $y$. (Technically, Otto works with one-sorted structures, so his stable coloring also has a convention for the relative ordering of $A$ and $B$, say $a \prec b$ whenever $a \in A$ and $b \in B$. This technicality will not matter in the following.)

By [15, Theorems 2.23 and 2.25], the stable coloring is definable in the $\operatorname{logic} C_{\infty, \omega}^{2}$, and its equivalence classes, our $A_{i}$ 's and $B_{j}$ 's, are exactly the equivalence classes with respect to $C_{\infty, \omega}^{2}$-equivalence. Therefore, the invariant $I_{C^{2}}$ as defined in [15, Section 3.2] encodes all the following information (plus more information that we won't need):

- The blocks $A_{i}$ and $B_{j}$, regarded as points.

- The linear ordering of these blocks.

- For each pair of blocks $A_{i}$ and $B_{j}$ whether there is an $R$-edge joining them.

- The cardinality of each block.

Recall from Section 2 that, for the logic FP+Card, structures $\mathfrak{A}$ (like our graphs $\langle A, B, R\rangle)$ are enriched with a new sort containing the natural numbers from 0 up to and including the size of $\mathfrak{A}$, with the standard successor function, and the resulting structure is called $\mathfrak{A}^{*}$. The standard linear ordering of natural numbers is easily FP-definable in $\mathfrak{A}^{*}$.

According to [15, Lemma 4.14(ii)], the invariant $I_{C^{2}}$ of $\mathfrak{A}$ is $\mathrm{FP}+$ Card interpretable in $\mathfrak{A}^{*}$, as a structure on the new, numerical sort. This means that the linear ordering of the blocks of $I_{C^{2}}$ is used to replace these blocks by numbers, the rest of the structure of $I_{C^{2}}$ is transferred to this copy, and the result is $\mathrm{FP}+$ Card-definable in $\mathfrak{A}^{*}$.

Once we have this form of $I_{C^{2}}$, we essentially have the structure that we called $\left\langle A^{\prime}, B^{\prime}, R^{\prime}\right\rangle$ in our description of the algorithm in Subsection 3.3. We can take $A^{\prime}$ to be the set of pairs $(0, i, r)$ where $i$ is the number representing a block $A_{i}$ and $r<\left|A_{i}\right|$, and we can take $B^{\prime}$ to be the set of pairs $(1, j, s)$ where $j$ represents a block $B_{j}$ and $s<\left|B_{j}\right| . R^{\prime}$ joins $(0, i, r)$ to $(1, j, s)$ if there was an $R$-edge from $A_{i}$ to $B_{j}$ - information that we saw is available in $I_{C^{2}}$.

Thus, we have a copy of $\left\langle A^{\prime}, B^{\prime}, R^{\prime}\right\rangle$, with linearly ordered underlying set (since it's in the numerical sort), FP+Card-definable in $\langle A, B, R\rangle^{*}$. To apply 
the path algorithm to this copy is to apply a polynomial time algorithm to an ordered structure. So the result, the decision whether there is a matching, is expressible in FP+Card, in fact in just FP, over this copy. Therefore, the decision is expressible in $\mathrm{FP}+\mathrm{Card}$ over $\langle A, B, R\rangle^{*}$, as claimed.

Remark 8 It is natural to ask whether, when a bipartite graph does not admit a complete matching, one can compute in $\tilde{\mathrm{CPT}}+$ Card the size of the largest (incomplete) matching.

Our definition above (and in [4]) of BGS programs allowed only Boolean output, so technically one cannot compute in $\tilde{\mathrm{CPT}}+$ Card anything other than Boolean queries. But this restriction in the definition was only a matter of convenience. The BGS computation model and thus the complexity classes $\tilde{\mathrm{CPT}}$ and $\tilde{\mathrm{CPT}}+$ Card can and should be extended to allow non-Boolean output whenever this is useful.

Once this extension is made, it is easy to show that the size of the largest matching in a bipartite graph is computable in $\tilde{\mathrm{CPT}}+$ Card. Indeed, $\langle A, B, R\rangle$ has a matching whose domain contains all but $s$ elements of $A$ if and only if there is a complete matching in the graph obtained by adding $s$ new elements to $B$ and enlarging $R$ so as to relate all elements of $A$ to the $s$ newly added elements.

The algorithm presented in this section depends on the fact that we deal with a bipartite graph. The notion of matching makes sense more generally. In any undirected, loopless graph, a matching is a family of edges no two of which have a common endpoint. A matching is complete is every vertex is incident to an edge from the matching. Whether a given graph has a complete matching can be decided in polynomial time by a variant of the path algorithm. But we do not know whether this decision can be computed without using choices or an ordering.

Question 9 Is the existence of complete matchings in general (nonbipartite) graphs computable in $\tilde{\mathrm{CPT}}+$ Card?

\section{Cai-Fürer-Immerman Graphs}

In describing the Cai, Fürer, Immerman construction, we follow, with a minor modification, Otto's presentation [15, Example 2.7], which is itself a minor modification of the presentation in [7]. 
Let $G$ be a finite connected graph; we shall need only the special case where $G$ is the complete graph on some number $m+1$ of points, but the construction is no harder to present in the general case. For each vertex $v$ of $G$, let $E_{v}$ be the set of edges incident with $v$. Fix a linear ordering $\preceq$ of the vertices of $G$. Using $G$, we define a new graph $G^{*}$ as follows. Each vertex of degree $d$ in $G$ gives rise to $2^{d}$ vertices of $G^{*}$ and each edge of $G$ gives rise to two vertices of $G^{*}$. Specifically, we let the vertices of $G^{*}$ be

- pairs $(v, X)$ where $v$ is a vertex of $G$ and $X$ is a subset of $E_{v}$, and

- pairs $(e,+)$ and $(e,-)$ where $e$ is an edge of $G$.

For each vertex $v$ of $G$, we write $U(v)$ for the set of associated vertices $(v, X)$ of $G^{*}$, and similarly for each edge $e$ of $G$, we write $U(e)$ for the pair of associated vertices $(e, \pm)$. (For vertices $(v, X) \in U(v)$, we chose to use, as members of the second component $X$, edges $\{v, w\} \in E_{v}$ rather than simply the distant vertices $w$ of those edges. The main reason for this choice is to match a visualization in which $U(v)$ is given in terms of "local data" at $v$ if edges are viewed as line segments.)

The edges of $G^{*}$ are also of two sorts; whenever edge $e$ and vertex $v$ are incident in $G$, we

- join $(v, X)$ to $(e,+)$ if $e \in X$, and

- join $(v, X)$ to $(e,-)$ if $e \notin X$.

In addition, we transport the linear ordering $\preceq$ of the vertices of $G$ to a preordering, also called $\preceq$, on the vertices of the form $(v, X)$ in $G^{*}$; that is, we put $(v, X) \preceq\left(v^{\prime}, X^{\prime}\right)$ just in case $v \preceq v^{\prime}$.

Before proceeding with the construction, it is useful to analyze the automorphisms of the structure (graph with a linear pre-ordering of some of the vertices) $\mathfrak{G}^{*}=\left\langle G^{*}, \preceq\right\rangle$. Preserving $\preceq$, such an automorphism $\alpha$ must map each $U(v)$ into itself. Then, to preserve adjacency, it must map each $U(e)$ into itself, for vertices in different $U(e)$ 's have neighbors in different $U(v)$ 's. Thus, $\alpha$ gives rise to a subset $S$ of the edge set of $G$, namely

$$
S=\{e: \alpha \text { interchanges }(e,+) \text { and }(e,-)\} .
$$

Obviously, $S$ determines the action of $\alpha$ on vertices of the form $(e, \pm)$. In fact, it completely determines $\alpha$, for a vertex $(v, X)$ is determined by which 
$(e, \pm)$ 's are adjacent to it. More formally, we have

$$
\alpha(e, \pm)=\left\{\begin{array}{ll}
(e, \mp) & \text { if } e \in S \\
(e, \pm) & \text { if } e \notin S
\end{array} \text { and } \quad \alpha(v, X)=\left(v, X \triangle\left(S \cap E_{v}\right)\right)\right.
$$

(As before, $\triangle$ denotes symmetric difference.) Conversely, for any set $S$ of edges of $G$, the preceding formulas define an automorphism of $\mathfrak{G}^{*}$.

The Cai, Fürer, Immerman graphs are subgraphs of $G^{*}$ obtained as follows. For any subset $T$ of the vertex set of $G$, let $G^{T}$ be the induced subgraph of $G^{*}$ containing all the vertices of the form $(e, \pm)$ but containing $(v, X)$ only if either $v \in T$ and $|X|$ is odd or $v \notin T$ and $|X|$ is even. An analysis exactly like that in the preceding paragraph shows that any automorphism of any $\mathfrak{G}^{T}$ (meaning of course $\left\langle G^{T}, \preceq\right\rangle$ ) and in fact any isomorphism from one $\mathfrak{G}^{T}$ to another $\mathfrak{G}^{T^{\prime}}$ must be given by the formulas above, for some set $S$ of edges of $G$. To describe which $S$ 's give isomorphisms between which $\mathfrak{G}^{T}$ 's, it is convenient to use the notation

$$
\operatorname{Odd}(S)=\{v: \text { The number of edges in } S \text { incident to } v \text { is odd. }\} \text {. }
$$

Then the $\alpha$ associated to $S$ maps $G^{T}$ to $G^{T^{\prime}}$ just in case $T \triangle T^{\prime}=\operatorname{Odd}(S)$ (equivalently, $T^{\prime}=T \triangle \operatorname{Odd}(S)$ ).

At this point we must recall two well-known facts from graph theory. The first is that $\operatorname{Odd}(S)$ always has even cardinality. Indeed, the total number of incident point-edge pairs $(v, e)$ is even because every edge $e$ contributes two such pairs. But, classifying the same pairs according to their vertex components $v$, we find that the number of these pairs is $\sum_{v} \operatorname{degree}(v)$. Modulo two, this sum is congruent to the number of odd summands, i.e., to the cardinality of $\operatorname{Odd}(S)$. So this cardinality must, like the sum, be even.

The second fact to recall is that for connected graphs, like our $G$, there is a converse to the first fact: Any set consisting of an even number of vertices is $\operatorname{Odd}(S)$ for some set $S$ of edges. To see this, pair off the vertices in the given set (arbitrarily) and choose for each pair a path joining them. Of course, if $P$ is the set of edges of one of these paths, then $\operatorname{Odd}(P)$ consists just of the two endpoints of that path. Summing up this information over all the chosen paths $P$ and reducing modulo two, we find that our given set of vertices is $\operatorname{Odd}(S)$ where $S$ consists of those edges that occur in an odd number of the paths $P$.

Applying these facts to our situation, we see that $\mathfrak{G}^{T}$ and $\mathfrak{G}^{T^{\prime}}$ are isomorphic if and only if $|T|$ and $\left|T^{\prime}\right|$ have the same parity. We write $\mathfrak{G}^{0}$ (resp. 
$\left.\mathfrak{G}^{1}\right)$ for $\mathfrak{G}^{T}$ when $|T|$ is even (resp. odd). Thus, $\mathfrak{G}^{0}$ and $\mathfrak{G}^{1}$ are well-defined up to isomorphism and are not isomorphic to each other.

In fact, $\mathfrak{G}^{0}$ and $\mathfrak{G}^{1}$ can be distinguished by the following simple property. In $G^{0}$ it is possible to choose one from each pair of vertices $(e, \pm)$ corresponding to an edge of $G$, in such a way that each block $U(v)$ contains a vertex $(v, X)$ adjacent to the chosen vertices in all the pairs $U(e)$ for $e$ adjacent to $v$ in $G$. This is easiest to see if we think of $G^{0}$ as $G^{\varnothing}$; then we simply choose $(e,-)$ from every $U(e)$. (If we think of $G^{0}$ as $G^{T}$ for some other $T$ of even size, then we should fix an $S$ with $\operatorname{Odd}(S)=T$, and we should choose $(e,+)$ if and only if $e \in S$.) On the other hand, no such choice is possible in $G^{1}$. Indeed, let us represent $G^{1}$ as $G^{T}$ for a specific $T$ of odd size, and suppose a successful choice of $(e, \pm)$ 's had been made. Let $S$ be the set of edges $e$ where the choice was $(e,+)$. Then we would have, for each vertex $v$ of $G$, that $\left(v, S \cap E_{v}\right) \in G^{T}$, which means that

$$
v \in T \Longleftrightarrow\left|S \cap E_{v}\right| \text { is odd } \Longleftrightarrow v \in \operatorname{Odd}(S) \text {. }
$$

So $T=\operatorname{Odd}(S)$, which is absurd as $|T|$ is odd and $|\operatorname{Odd}(S)|$ is even.

Let us now specialize to the case where $G$ is the complete graph on $m+1$ vertices. Let $\mathfrak{H}_{m}^{0}$ and $\mathfrak{H}_{m}^{1}$ be padded versions of $\mathfrak{G}^{0}$ and $\mathfrak{G}^{1}$, obtained by adjoining $2^{m^{2}}$ isolated vertices, not in the field of the pre-ordering $\preceq$.

Proposition 10 There is a polynomial time BGS program that accepts $\mathfrak{H}_{m}^{0}$ and rejects $\mathfrak{H}_{m}^{1}$ for all $m$.

Proof The program checks whether there is a choice of one vertex $(e, \pm)$ from each $U(e)$ such that each $U(v)$ contains a vertex whose neighbors were all chosen. We saw above that such a choice is possible in $\mathfrak{H}_{m}^{0}$ but not in $\mathfrak{H}_{m}^{1}$. To write the program in BGS, think of it as consisting of two phases. In the first phase, it goes through all the blocks $U(e)$ in order (i.e., in the order induced on edges $e$ by $\preceq$ ), splitting into parallel subcomputations each of which has one choice of vertices from the $U(e)$ 's. Then in the second phase, each of these subcomputations goes through the $U(v)$ 's in order, checking whether there is a vertex whose neighbors were all chosen.

The number of edges in $G$ is $(m+1) m / 2 \leq m^{2}$. So the number of parallel subcomputations is no bigger than $2^{m^{2}}$. The padding in the definition of $\mathfrak{H}_{m}^{i}$ ensures that this is a polynomial in the input size. It easily follows that a PTime version of this BGS program does what is required of it in the proposition. 
In contrast, Lemma 2.8 of [15] (see also Corollary 7.1 of [7]) shows that $\mathfrak{H}_{m}^{0}$ and $\mathfrak{H}_{m}^{1}$ cannot be distinguished by any sentence in $C_{\infty, \omega}^{m}$. (Our graphs differ from Otto's in two ways. Where we have a single vertex $(e,+)$ or $(e,-)$ joined to vertices in two $U(v)$ 's corresponding to the two endpoints of $e$, he has two adjacent vertices, each joined to vertices in just one $U(v)$. And his graphs are not padded with isolated vertices. Neither of these differences affects the proof that the graphs are $C_{\infty, \omega}^{m}$-equivalent.)

Thus, these examples of Cai, Fürer, and Immerman, with sufficient padding, show that FP+Card does not include $\tilde{\mathrm{CPT}}$; so $\tilde{\mathrm{CPT}}+$ Card properly includes FP+Card.

Since the padding looks very artificial, it is natural to ask what happens if we omit it. Writing $\mathfrak{G}_{m}^{i}$ for $\mathfrak{G}^{i}$ in the special case where $G$ is a complete graph on $m+1$ vertices, we still have that $\mathfrak{G}_{m}^{0}$ and $\mathfrak{G}_{m}^{1}$ are $C_{\infty, \omega}^{m}$-equivalent, just as before. But the proof of Proposition 10 breaks down, since the computation is no longer PTime bounded. The input structures $\mathfrak{G}_{m}^{i}$ have size only $(m+$ 1) $\left(2^{m-1}+m\right)$, so polynomial time would mean time bounded by $2^{\mathrm{cm}}$ for some $c$. This is insufficient for generating all the choices of $(e, \pm)$ 's. We do not know whether $\tilde{\mathrm{CPT}}$ or even $\tilde{\mathrm{CPT}}+$ Card can distinguish all the $\mathfrak{G}_{m}^{0}$ 's from the $\mathfrak{G}_{m}^{1}$ 's, but an argument from the proof of Corollary 7.1 of [0] can be adapted to give the following result.

Proposition 11 The isomorphism closure of the class $\left\{\mathfrak{G}_{m}^{0}: m \in \mathbb{N}\right\}$ is in PTime.

Proof We must exhibit a PTime algorithm which, given a structure $\mathfrak{X}$ of the appropriate vocabulary and given an ordering of its underlying set, decides whether $\mathfrak{X} \cong \mathfrak{G}_{m}^{0}$ for some $m$.

It is straightforward to check whether $\mathfrak{X} \cong \mathfrak{G}_{m}^{i}$ for some $m$ and some $i$ : First count the number of vertices and use it to compute $m$. Then check whether $\preceq$ is a linear pre-ordering with $m+1$ equivalence classes $U(v)$, each of size $2^{m-1}$. Then check whether the remaining vertices come in pairs $U(e)$, one for each pair of $v$ 's. Label the vertices in each pair $U(e)$ with + and - , say using + for the earlier one in the given ordering of the set of vertices. Then label each vertex in each $U(v)$ by the sequence of + 's and -'s describing which vertices from the $U(e)$ 's it is adjacent to. (We can do this, with sequences because of the ordering of vertices.) Then check whether the sequences associated to any two distinct vertices from the same $U(v)$ differ 
in a nonzero even number of locations. $\mathfrak{X}$ has the form $\mathfrak{X} \cong \mathfrak{G}_{m}^{i}$ if and only if all these computations and checks succeed.

It remains to distinguish $\mathfrak{G}_{m}^{0}$ from $\mathfrak{G}_{m}^{1}$. This is done by a slight variant of the approach used above in the padded case (Proposition 10). From each pair $(e, \pm)$ choose the vertex labeled $(e,-)$ above. Label a block $U(v)$ "good" if it contains a vertex adjacent only to chosen vertices and "bad" otherwise. Of course, if all blocks are good, then our graph $\mathfrak{X}$ is, up to isomorphism, $\mathfrak{G}_{m}^{0}$.

The same holds if the number of bad blocks is even. Indeed, in this case, there is a set $S$ of edges of the complete graph $G$ such that $\operatorname{Odd}(S)$ is exactly

the set of vertices corresponding to bad blocks. If we choose $(e,+)$ instead of $(e,-)$ at the edges $e \in S$, then the new choices have the property that every $U(v)$ contains a vertex adjacent only to chosen ones.

On the other hand, if the number of bad blocks is odd, then a similar argument shows that $\mathfrak{X} \cong \mathfrak{G}_{m}^{1}$.

It remains to observe that we can determine in PTime which blocks are bad and (thanks to the ordering) how many of them there are. So this algorithm works in PTime and accepts precisely (the isomorphs of) the graphs $\mathfrak{G}_{m}^{0}$.

Question 12 Can $\tilde{\mathrm{CPT}}+$ Card (or even $\tilde{\mathrm{CPT}}$ ) distinguish $\mathfrak{G}_{m}^{0}$ from $\mathfrak{G}_{m}^{1}$ for all $m$ ?

If the answer is negative, then we have a separation of PTime from $\tilde{\mathrm{CPT}}+\mathrm{Card}$. If the answer is affirmative, then we merely have another separation of $\tilde{\mathrm{CPT}}+$ Card from $\mathrm{FP}+$ Card, which we already had using $\mathfrak{H}_{m}^{i}$. The new separation would be aesthetically preferable, since it avoids padding.

\section{$5 \quad$ Multipedes}

In this section, we study Boolean queries concerning the multipedes introduced in [13]. We begin by recalling the relevant definitions and results from [13. That paper uses five notions of $k$-multipede, for $k=1,2^{-}, 2,3,4$. We shall not need the first two of these, so we begin with 2-multipedes.

Definition 13 A 2-multipede is a finite 2-sorted structure, the two sorts being called "segments" and "feet," with the following data. 
- A function $S$ from feet to segments, such that every segment is the image of exactly two feet.

- A family of 3-element sets of segments, called "hyperedges." (This family is coded as a totally irreflexive and symmetric ternary relation.)

- A family of 3-element sets of feet, called "positive triples," (similarly coded).

These data are subject to the following requirements.

- If $P$ is a positive triple of feet, then its image $S(P)$ is a hyperedge. In particular, $S$ is one-to-one on $P$.

- If $H$ is a hyperedge then, of the eight triples of feet that $S$ maps onto $H$, exactly four are positive.

- If $P$ and $P^{\prime}$ are positive triples of feet with $S(P)=S\left(P^{\prime}\right)$, then $\left|P \triangle P^{\prime}\right|$ is even.

Notice that, for any three-element set $H$ of segments, there are exactly eight three-element sets of feet mapped onto $H$ by $S$. These eight are partitioned into two sets of four by the equivalence relation "even symmetric difference." The positivity relation picks out one of these two equivalence classes for each hyperedge $H$.

Definition 14 A 3-multipede is a 2-multipede together with a linear ordering $\leq$ of the set of all segments.

Definition 15 A 4-multipede is a 3-multipede together with a third sort, called "sets," and a binary relation $\varepsilon$ between segments and sets such that every set (in the ordinary sense) of segments is $\{s: s \varepsilon x\}$ for a unique set (in the sense of the structure) $x$.

In other words, up to isomorphism, the sort of sets is exactly the power set of the sort of segments and $\varepsilon$ is the membership relation.

Definition 16 A multipede is odd if, for every nonempty set $X$ of segments, there is a hyperedge whose intersection with $X$ has odd cardinality. 
The value of oddness and of the linear ordering in the definition of 3multipedes is the following result, combining Lemmas 4.1 and 4.4 of [13.

Proposition 17 (《13]) Odd 3-multipedes and odd 4-multipedes are rigid.

Proof Consider first an automorphism $\alpha$ of an odd 3-multipede. Because of the linear ordering, it must fix every segment. So all it can do with feet is to interchange the two feet in $S^{-1}(\{s\})$ for certain segments $s$. Let $X$ be the set of segments $s$ whose two feet $\alpha$ interchanges. Since $\alpha$ preserves positivity of triples of feet, the intersection of $X$ with each hyperedge must have even cardinality. Since the multipede is odd, this means $X=\varnothing$, and so $\alpha$ fixes all feet.

In the case of a 4-multipede, we see as above that an automorphism fixes all segments and all feet. In order to preserve $\varepsilon$, it must also fix all sets.

The main work in 13] involves the notion of a $k$-meager multipede (for $k \in \mathbb{N}$ ); we omit the definition here because we shall avoid needing it. We do need two trivial (given the definition) and two deep properties of meagerness. The trivial properties are that meagerness depends only on the segments and hypergraphs and that $k$-meagerness implies $l$-meagerness for all $l<k$. The deep properties are the following two results from [13]; the first is Theorem 3.1 and the second combines Lemmas 4.2 and 4.5 of [13].

Proposition 18 (《13) For any positive integers $l \geq 2$ and $N$, there exists an odd, l-meager multipede with more than $N$ segments.

We observe that it doesn't matter in this proposition whether "multipede" refers to 2-, 3-, or 4-multipedes. Once we have an odd, $l$-meager 2-multipede, we can expand it with an arbitrary linear ordering of its segments and we can adjoin an appropriate universe of sets to get an odd, $l$-meager 4-multipede.

Proposition 19 (《13]) No formula of $C_{\infty, \omega}^{l}$ can distinguish between the two feet of any segment in an l-meager multipede.

The purpose of these results in 13] was to exhibit a finitely axiomatizable (in first-order logic) class of structures, namely the odd 4-multipedes, such that all structures in the class are rigid but no $C_{\infty, \omega}^{\omega}$ formula can define a linear ordering on all structures of the class. The addition of sets, in going from 3-multipedes to 4-multipedes, served to make "odd" first-order definable. 
In the present paper, our interest is in definability (or computability) of Boolean queries, not linear orderings. To apply the ideas of [13] in this context, we make one additional definition, intended to apply to 2-, 3,- and 4-multipedes simultaneously.

Definition 20 A multipede with a shoe is a multipede with a distinguished foot, called the "foot with a shoe" or simply the "shoe." In the case of 3- and 4-multipedes, it is further required that $S$ of the shoe is the first segment in the order $\leq$.

The first Boolean query we shall consider is the isomorphism problem for 4-multipedes with shoes. The input here is a pair of 4-multipedes, each with a shoe. (Since 4-multipedes are 3-sorted structures, it is convenient to regard a pair of them as a 6-sorted structure.) The question is whether the two are isomorphic.

Theorem 21 The isomorphism problem for 4-multipedes with shoes is in $\tilde{C} P T$.

Proof Since the segments of a 4-multipede are linearly ordered, any isomorphism is uniquely determined on segments and therefore on sets, and it is easy to check in choiceless polynomial time whether the hyperedges in the two multipedes match up properly. The only real problem is whether the feet can be matched up so as to preserve $S$ and positivity.

If the input multipedes have $n$ segments each, then there are $2^{n}$ ways to match up the feet while preserving $S$, since for each of the $n$ pairs of corresponding segments in the two multipedes, there are two ways to match up their feet. The problem is whether any of these $2^{n}$ matchings preserves positivity.

Because of the universe of sets in a 4-multipede, the input structures are larger than $2^{n}$. So a PTime bounded BGS algorithm has enough time to construct, in parallel, all the relevant matchings of feet and to check whether any of them preserve positivity.

In this proof, the role of the sets is to serve as padding, making "polynomial time" long enough to carry out the algorithm. The only reason we didn't have to resort to explicit padding here (as we did in the case of the Cai, Fürer, Immerman examples above) is that the necessary padding was already done, for a different purpose, in [13]. Of course, this raises the 
question of what happens without padding, i.e., with 3-multipedes; we shall return to this question after the next result, which completes our discussion of 4-multipedes.

Theorem 22 The isomorphism problem for 4-multipedes with shoes is not in $C_{\infty, \omega}^{\omega}$ and therefore not in FP+Card.

Proof Suppose we had a sentence $\theta$ of $C_{\infty, \omega}^{\omega}$ expressing isomorphism between 4-multipedes with shoes. Fix $l$ so large that $\theta$ is in $C_{\infty, \omega}^{l}$, and let $M$ be an odd, l-meager 4-multipede, which exists by Proposition 18. Let $\mathfrak{M}_{0}$ and $\mathfrak{M}_{1}$ be the two expansions of $M$ with shoes, i.e., one of the two feet of the first segment is the shoe in $\mathfrak{M}_{0}$ and the other is the shoe in $\mathfrak{M}_{1}$. By Proposition 17, $\mathfrak{M}_{0}$ and $\mathfrak{M}_{1}$ are not isomorphic, for an isomorphism would be a non-trivial automorphism of $\mathfrak{M}$. Thus, $\theta$ must be false in the structure $\mathfrak{M}_{0}+\mathfrak{M}_{1}$ but true in $\mathfrak{M}_{0}+\mathfrak{M}_{0}$.

This means that the Spoiler has a winning strategy in $C^{l}$ game for the pair of structures $\mathfrak{M}_{0}+\mathfrak{M}_{1}$ and $\mathfrak{M}_{0}+\mathfrak{M}_{0}$. (See [15, Theorem 2.1].) We obtain a contradiction by exhibiting a winning strategy for the Duplicator in this game.

The proofs of Lemmas 4.2 and 4.5 in 13 provide a winning strategy for the Duplicator in the $C^{l}$ game for the pair of structures $\mathfrak{M}_{0}$ and $\mathfrak{M}_{1}$. And the Duplicator has a trivial winning strategy for the pair $\mathfrak{M}_{0}$ and $\mathfrak{M}_{0}$ : just copy whatever the Spoiler does. Combining these two known strategies, we get a winning strategy for the Duplicator for the pair $\mathfrak{M}_{0}+\mathfrak{M}_{1}$ and $\mathfrak{M}_{0}+\mathfrak{M}_{0}$ as follows. When Spoiler picks a subset of one of these structures, think of it as two subsets, one in each of the two component multipedes. Apply the known strategies to find two subsets of the same cardinalities in the component multipedes of the other board, and play the union of these two subsets. Then, when Spoiler picks a point in one of these subsets, pick a point on the other board by consulting the appropriate one of the known strategies.

In effect, Duplicator is playing the $C^{l}$ game for $\mathfrak{M}_{0}+\mathfrak{M}_{1}$ and $\mathfrak{M}_{0}+\mathfrak{M}_{0}$ by playing separately the trivial game for the first components $\mathfrak{M}_{0}$ and $\mathfrak{M}_{0}$ and the game for the second components $\mathfrak{M}_{0}$ and $\mathfrak{M}_{1}$. Since he wins in both components, he also wins the overall game.

The last two theorems give us, once again, a $\tilde{\mathrm{CPT}}$ computable query that goes beyond FP+Card. 
Turning to 3-multipedes, we see that Theorem 22 remains true with the same proof. But the proof of Theorem 21 no longer applies, because a 3multipede with $n$ segments has only $3 n$ elements and so polynomial time is inadequate for producing all possible matchings of the feet. As a result, we do not know whether isomorphism of 3-multipedes with shoes is in $\tilde{\mathrm{CPT}}$ or even in $\tilde{\mathrm{CPT}}+$ Card. But we do have the following weaker result.

Theorem 23 Isomorphism of 3-multipedes with shoes is computable in PTime.

Proof We must present a PTime algorithm which, given a structure $\mathfrak{A}+\mathfrak{B}$ where $\mathfrak{A}$ and $\mathfrak{B}$ are 3 -multipedes with shoes, and given a linear ordering $\preceq$ of their union, decides whether they are isomorphic. There is a slight possibility of confusion between the different orderings here, the linear orderings of segments that are part of the 3-multipede structure of $\mathfrak{A}$ and $\mathfrak{B}$, and the additional ordering $\preceq$ of the whole combined structure. The latter will be used only to distinguish between the two feet of any segment (in either component multipede); we'll call one the left and the other the right foot. We fix the terminology so that the left foot is $\prec$ the right except that in both multipedes the shoe is declared to be the left foot of its segment regardless of what $\preceq$ does. For the rest of the proof, any mention of an ordering refers to the orderings of segments that are part of the 3-multipede structure.

As in the proof of Theorem 21, thanks to the orderings of segments, there is no difficulty deciding whether the hypergraph structures on the segments agree. The problem is to decide whether the feet can be matched appropriately. If there are $n$ segments then there are $2^{n}$ possible (i.e., respecting the function $S$ ) matchings, and the algorithm lacks the time to check each one to see if it preserves positivity. Instead, let the algorithm proceed as follows.

Take one specific matching $\mu$, namely the one that maps left feet to left feet and (therefore) right feet to right feet (of the corresponding segments, of course). If it happens to preserve positivity, then output "yes" (or ignore this obvious answer and proceed as in the general case). For each of the two multipedes $\mathfrak{A}$ and $\mathfrak{B}$, list all its hyperedges in lexicographic order (with respect to the ordering of segments). Of course, the two multipedes have the same number, say $m$, of hyperedges; otherwise, the algorithm would have detected non-isomorphism earlier and we wouldn't be looking for a matching of the feet. Form an m-component vector $\vec{v}$ of 0 's and 1's, where the $k^{\text {th }}$ 
entry is 0 if $\mu$ preserves positivity of triples of feet at the $k^{\text {th }}$ hyperedge and 1 otherwise.

Any other possible matching is obtained from $\mu$ by reversals at some set $X$ of segments of $\mathfrak{A}$. Call the result $\mu_{X}$. (So $\mu=\mu_{\varnothing}$.) In order for $\mu_{X}$ to be an isomorphism, i.e., to preserve positivity at all segments, $X$ must have an odd intersection with those hyperedges where $\mu$ failed to preserve positivity and an even intersection with the other hyperedges. We reformulate this criterion as follows. Represent any $X$ by an $n$-component vector of zeros and 1 's, where the $k^{\text {th }}$ entry is 1 if and only if the $k^{\text {th }}$ segment is in $X$. Also, let $A$ be the segment-hyperedge adjacency matrix; it is the $m \times n$ matrix whose $(k, l)$ component is 1 if the $k^{\text {th }}$ hyperedge contains the $l^{\text {th }}$ segment. Then the condition for $\mu_{X}$ to preserve positivity is simply that $A \vec{x}=\vec{v}$, where both $\vec{x}$ and $\vec{v}$ are considered as column vectors, and where arithmetic is done modulo 2 .

Thus, the isomorphism question is reduced to the question of solvability of a system of linear equations $A \vec{x}=\vec{v}$ over the field $\mathbb{Z} / 2$. But such questions are easily solved in polynomial time, by Gaussian elimination.

We repeat the main question left open by the results in this section.

Question 24 Is isomorphism of 3-multipedes with shoes computable in $\tilde{\mathrm{CPT}}+$ Card or in $\tilde{\mathrm{CPT}}$ ?

A negative answer to the $\tilde{\mathrm{CPT}}+$ Card version of the question would separate PTime from $\tilde{\mathrm{CPT}}+\mathrm{Card}$. A positive answer would only give yet another separation of $\tilde{\mathrm{CPT}}+$ Card from $\mathrm{FP}+\mathrm{Card}$ (without any unpleasant padding).

\section{Determinants}

The use of linear algebra modulo 2 in the proof of Theorem 23 suggests that this topic or more generally linear algebra over finite fields may lead to interesting problems at or near the border between PTime and $\tilde{\mathrm{CPT}}+$ Card. In this section, we consider problems of this sort, related to computing determinants or at least deciding whether a given matrix has zero determinant.

\subsection{Matrices and Determinants}

The method of Gaussian elimination, i.e., reducing a matrix to echelon form by row or column operations, computes determinants of $n \times n$ matrices in 
$O\left(n^{3}\right)$ arithmetical operations. When the matrix entries come from a fixed finite field (or commutative ring), this observation shows that determinants are computable in polynomial time. (If the matrix entries come from an infinite field or ring, then one must take into account how the entries are presented and how complex the arithmetical operations are. We shall discuss the infinite case briefly below.)

Matrices are usually regarded as having their rows and columns given in a specified order, and the Gaussian elimination algorithm makes use of this order in deciding which row operations to apply. Our concern in this section will be with "matrices" in which the rows and columns are indexed by unordered sets; thus Gaussian elimination cannot be used. We use matrices as inputs to computations, so, in accordance with the conventions of BGS, we shall code matrices as structures.

There are two inequivalent ways to make precise the notion of a matrix with unordered rows and columns.

Definition 25 Let $I$ and $J$ be finite sets, and let $R$ be a finite commutative ring. An $I \times J$ matrix with entries from $R$ is a function $M: I \times J \rightarrow R$. We regard $M$ as a two-sorted structure, the sorts being $I$ and $J$, with basic relations

$$
M_{r}=\{(i, j) \in I \times J: M(i, j)=r\}
$$

for all $r \in R$.

Definition 26 Let $I$ be a finite set, and let $R$ be a finite commutative ring. An $I$-square matrix with entries from $R$ is a function $M: I \times I \rightarrow R$. We regard $M$ as a one-sorted structure with underlying set $I$ and with basic relations

$$
M_{r}=\{(i, j) \in I \times I: M(i, j)=r\}
$$

for all $r \in R$.

An alternative but equivalent way to code matrices as structures would be to include $R$ as an additional sort, to have the matrix itself as a function $I \times J \rightarrow R$ or $I \times I \rightarrow R$, and to include in the vocabulary names for all members of $R$.

Notice that, even when $|I|=|J|$, an $I \times J$ matrix differs in an essential way from an $I$-square matrix. An ordering of (the structure representing) an 
$I \times J$ matrix independently orders both $I$ and $J$; an ordering of (the structure representing) an $I$-square matrix merely orders $I$.

Thus, an $I$-square matrix has a well-defined determinant in the following sense. If one linearly orders $I$ then one obtains a matrix in the usual sense. The determinant of this matrix is independent of the ordering because if one changes the ordering the effect is to permute the rows and the columns in the same way. If the row permutation is odd and therefore reverses the sign of the determinant, then the column permutation reverses the sign again, restoring the original value. In contrast, even when $|I|=|J|$, the determinant of an $I \times J$ matrix is defined only up to sign. One gets a square matrix in the usual sense by fixing any orderings of $I$ and $J$, but changing to different orderings may change the sign of the determinant.

We observe that the question whether a matrix has zero determinant makes good sense not only for $I$-square matrices but also for $I \times J$ matrices as long as $|I|=|J|$. Although the determinant is defined only up to sign, the sign doesn't matter if we only care whether the determinant is zero. Similarly, it makes good sense to speak of the rank of an $I \times J$ matrix (whether or not $|I|=|J|)$.

Remark 27 One can view an $I$-square matrix as an $I \times J$ matrix together with a specified bijection between $I$ and $J$. Every structure of the latter sort is isomorphic to one where $I=J$ and the specified bijection is the identity; if the isomorphism is required to be the identity on $I$ then it is unique.

\subsection{Determinants Modulo Two}

In this subsection, we consider determinants of square matrices with entries from the two-element field $\mathbb{Z} / 2$. For this particular field, an $I$-square matrix $M$ can be regarded as a directed graph with vertex set $I$ and arc set $M_{1}=\left\{(i, j) \in I^{2}: M(i, j)=1\right\}$, for the other relation, $M_{0}$, in the structure representing $M$ is then determined as the complement of $M_{1}$. In other words, any square matrix over $\mathbb{Z} / 2$ can be regarded as the incidence matrix of a directed graph. The graph here may have loops and may have pairs of opposite $\operatorname{arcs}(i, j)$ and $(j, i)$ but cannot have parallel arcs; an arc is simply an ordered pair of vertices.

Another simplification resulting from the restriction to $\mathbb{Z} / 2$ is that the problems "compute the determinant" and "is the determinant zero?" are equivalent, since there is only one possible non-zero value. We shall consider 
the problem in the form "is the determinant zero," for it is in this form that our results generalize to other finite fields.

A third simplification is that determinants are well-defined for $I \times J$ matrices with $|I|=|J|$. The sign ambiguity described earlier disappears in characteristic 2 where $x=-x$. Nevertheless, the algorithm presented in this subsection applies to $I$-square matrices only. From the point of view described in Remark 27, we shall make real use of the given bijection between the rows and the columns. Later, we shall consider ways to avoid this.

Theorem 28 The determinant of the square matrix over $\mathbb{Z} / 2$ represented by a finite directed graph is definable in FP+Card.

Here we identify the possible values 0 and 1 of the determinant with the truth values, so that the determinant becomes a Boolean query.

Proof We describe an algorithm for deciding whether any $I$-square matrix is non-singular. The algorithm is easily seen to be formalizable as a polynomial time algorithm in BGS+Card. Afterward we sketch how to convert the algorithm into a definition in FP+Card.

We begin with a preliminary observation. Given two $I$-square matrices $M$ and $N$, we can compute the product matrix $M N$, which is also an $I$-square matrix. Indeed, $(i, j)$ is an arc in the graph $M N$ if and only if the cardinality of the set

$$
\left\{k \in I:(i, k) \in M_{1} \text { and }(k, j) \in N_{1}\right\}
$$

is odd. Since the parity of a natural number (which may be regarded as a von Neumann ordinal - see Section 2) is easily in C̈PT, it follows that all entries of the product matrix can be computed in $\tilde{\mathrm{CPT}}+$ Card.

Next, we observe that we can compute powers of a matrix, even when the exponent is so large that it is given in binary notation. We first describe how binary notation for natural numbers can be handled in the BGS context.

The idea is that the binary representation of a natural number $r$, say of length $l=\lg (r)$, amounts to a subset $C$ of $\{0, \ldots, l-1\}$, namely the set of places where a 1 occurs in the binary notation. Thus $r=\sum_{c \in C} 2^{c}$. We remark that, for non-zero $r$ and therefore nonempty $C$, the largest element of $C$ is easily computable from $C$, namely as $\bigcup C$ (where natural numbers are identified with von Neumann ordinals).

Suppose we are given an $I$-square matrix $M$ and an integer $r$ in binary notation, i.e., the set $C$ as above. Then we can compute $M^{r}$ in time polynomial in $|I|$ and $\lg (r)$. The computation of $M^{r}$ is done by repeated squaring, 
i.e., by applying the recursion formulas

$$
M^{r}= \begin{cases}M & \text { if } r=1 \\ \left(M^{r / 2}\right)^{2} & \text { if } r \geq 2 \text { is even } \\ \left(M^{(r-1) / 2}\right)^{2} \cdot M & \text { if } r \geq 2 \text { is odd. }\end{cases}
$$

Here is a BGS program for this algorithm, using matrix multiplication as an "external" function, which means that for the complete algorithm one should replace all matrix multiplications here by the algorithm described above.

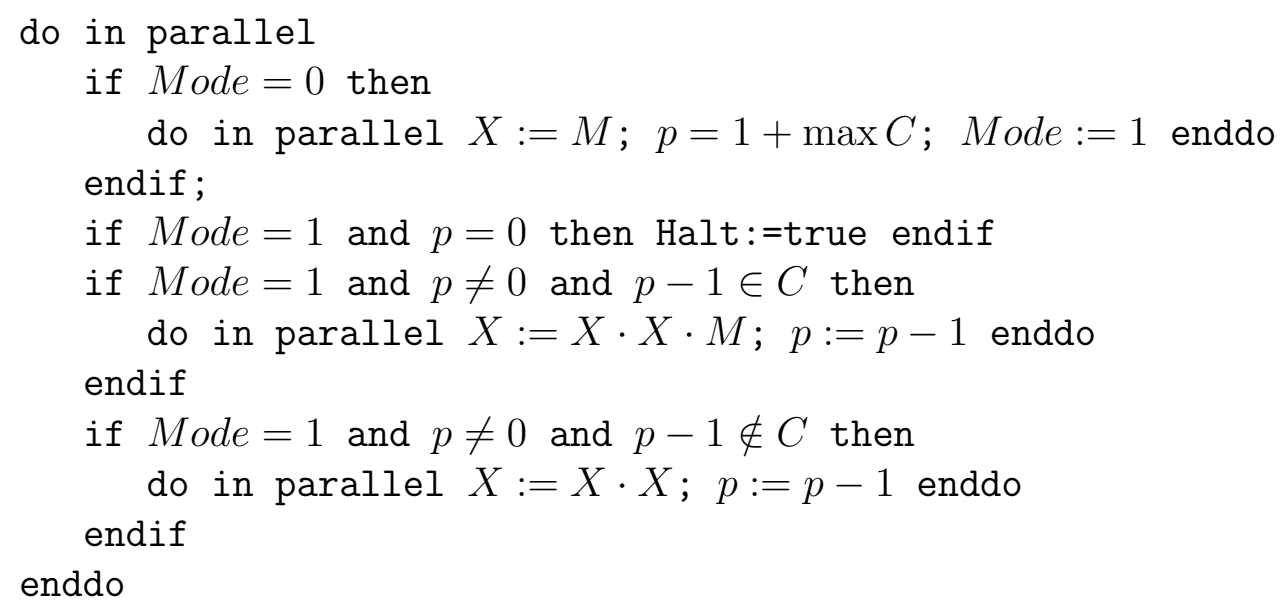

As a final preparatory step, we compute the order of $\mathrm{GL}_{n}(\mathbb{Z} / 2)$, the group of non-singular $n \times n$ matrices over $\mathbb{Z} / 2$. This order is

$$
g=\left(2^{n}-1\right)\left(2^{n}-2\right)\left(2^{n}-4\right) \cdots\left(2^{n}-2^{n-1}\right)=\prod_{i=0}^{n-1}\left(2^{n}-2^{i}\right) .
$$

To see this, we use the fact that an $n \times n$ matrix is non-singular if and only if its columns are linearly independent vectors in $(\mathbb{Z} / 2)^{n}$. If we imagine the columns being chosen one at a time, the first column of such a matrix can be any non-zero vector in $(\mathbb{Z} / 2)^{n}$; the second can be any vector different from the first and from 0 ; the third can be any vector that is not a linear combination of the first two; and in general any column can be any vector not a linear combination of the previously chosen columns. Thus, there are $2^{n}-1$ choices for the first column, each leaving $2^{n}-2$ choices for the second, each leaving $2^{n}-4$ choices for the third, and so on. 
Now given an $I$-square matrix $M$, in the form of a digraph with vertex set $I$, we can determine whether it is non-singular as follows in C PT+Card. First, use the cardinality function to determine the von Neumann ordinal $n=|I|$. From this, compute the group order $g$ in binary notation. Notice that $g<2^{n^{2}}$, so the length of this binary expansion (max $C$ in the notation above) is bounded by $n^{2}$. Our formula for $g$ above makes the computation of this binary expansion a simple matter, easily programmed in BGS (without further use of the cardinality function). Next, compute $M^{g}$; as indicated above, this can be done in $\tilde{\mathrm{CPT}}+$ Card. Finally, output 1 if $M^{g}$ is the identity matrix (i.e., if the arcs in the digraph $M^{g}$ are exactly the loops $(i, i)$ for all $i \in I$ ) and 0 otherwise.

To see that this algorithm gives the correct answer, recall from elementary group theory the fact (a special case of Lagrange's theorem) that the order of an element in a group always divides the order of the group. Thus, if $M$ is non-singular then the matrix obtained by ordering $I$ arbitrarily is an element of $\mathrm{GL}_{n}(\mathbb{Z} / 2)$, so its $g^{\text {th }}$ power is the identity matrix, and the same follows for $M$. If, on the other hand, $M$ is singular, then so are all its powers; in particular none of its powers is the identity matrix. This completes the proof that non-singularity of square matrices over $\mathbb{Z} / 2$ is computable in $\tilde{\mathrm{CPT}}+$ Card.

Finally, we briefly indicate why this algorithm yields a definition in $\mathrm{FP}+$ Card. Since the input is a structure (directed graph) of size $n, \mathrm{FP}+\mathrm{Card}$ works with a two-sorted structure $\mathfrak{A}^{*}$ consisting of the input graph and the natural numbers up to $n$. The algorithm above used natural numbers up to $n^{2}$ (to produce the binary expansion of $g$ ), but these can be coded as pairs of numbers below $n$. The computation of $g$ (in binary form) is a polynomial time algorithm working on a numerical input (the second sort of $\mathfrak{A}^{*}$ ), so it can be expressed in FP. The repeated squaring algorithm for computing $M^{g}$ can be cast as a definition, using the fixed-point operator, of the ternary relation

$$
\left\{(i, j, q):(i, j) \in M^{g_{q}}\right\}
$$

where $g_{q}$ means the integer represented by the $q$ most significant digits in the binary expansion of $g$. (More precisely, this is a quaternary relation because, as indicated above, $q$ is represented by a pair of elements of the numerical sort in $\mathfrak{A}^{*}$.) Finally, the comparison between $M^{g}$ and the identity matrix is expressible in first-order logic.

To complement the previous theorem, we show next that the cardinality 
function is essential in this or any choiceless algorithm for determinants over the two-element field.

Theorem 29 The determinant of the square matrix over $\mathbb{Z} / 2$ represented by a finite directed graph is not computable in $\tilde{C} P T$.

Proof Temporarily fix a positive integer $n$. As we saw in the proof of Theorem 28, the number of non-singular $n \times n$ matrices over $\mathbb{Z} / 2$ is

$$
g=\prod_{i=0}^{n-1}\left(2^{n}-2^{i}\right)
$$

Since the total number of $n \times n$ matrices over $\mathbb{Z} / 2$ is $2^{n^{2}}$, the probability that such a matrix, chosen uniformly at random, is non-singular is

$$
\frac{g}{2^{n^{2}}}=\prod_{i=0}^{n-1}\left(1-\frac{2^{i}}{2^{n}}\right)=\prod_{j=1}^{n}\left(1-\frac{1}{2^{j}}\right) .
$$

This product is therefore the probability that a random (with respect to the uniform distribution) directed graph on an $n$-element vertex set has, when viewed as a matrix, determinant 1 .

Now un-fix $n$ and let it tend to infinity. The asymptotic probability that a large, random, directed graph has determinant 1 is

$$
\prod_{j=1}^{\infty}\left(1-\frac{1}{2^{j}}\right)
$$

This infinite product is obviously strictly smaller than 1 . It is strictly greater than 0 (i.e., it converges in the conventional terminology) because the series $\sum_{j}\left(1 / 2^{j}\right)$ converges. (Recall the standard proof: $1-x>e^{-2 x}$ for all positive $x \leq \frac{1}{2}$. Apply this to $x=1 / 2^{j}$ and take the product over $j$, obtaining a convergent sum in the exponent.)

But the zero-one law proved by Shelah [16] (see also [3]) implies that any property of digraphs computable in $\tilde{\mathrm{CPT}}$ must have asymptotic probability 0 or 1 . Therefore, "determinant 1 " is not such a property. 


\subsection{Other Finite Fields}

The FP+Card definition of "non-singular" given in the preceding subsection for square matrices over $\mathbb{Z} / 2$ works, with minor modifications, over any finite field $F$. Of course, when the field has more than two elements, the "nonsingular" question is weaker than the problem of actually evaluating the determinant.

To indicate the minor modifications explicitly, let $F$ be a finite field of characteristic $p$ and cardinality $q=p^{e}$. Then to decide non-singularity of square matrices over $F$, we can use the algorithm described above for the special case $q=2$ with the following two changes. First, the order of the group $\mathrm{GL}_{n}(F)$ is

$$
g=\left(q^{n}-1\right)\left(q^{n}-q\right)\left(q^{n}-q^{2}\right) \ldots\left(q^{n}-q^{n-1}\right)=\prod_{i=0}^{n-1}\left(q^{n}-q^{i}\right)
$$

i.e., $q$ replaces 2 in the earlier formula.

Second, multiplying matrices becomes slightly more tedious but remains straightforward. Given two $I$-square matrices $M$ and $N$, to compute the $(i, j)$ entry of a product matrix $M N$, first do the following for each element $z \in F$. Consider the set $P_{z} \subseteq F^{2}$ of pairs $(x, y)$ whose product in $F$ is $z$. Since $F$ is fixed in this discussion, our BGS program or FP+Card formula can contain a complete listing of all the $P_{z}$ 's. Use the cardinality function to obtain the numbers

$$
m_{z}=\left|\left\{k \in I:(M(i, k), N(k, j)) \in P_{z}\right\}\right|
$$

and then, in a trivial polynomial time computation, reduce these numbers modulo $p$ to obtain $\bar{m}_{z}=m_{z} \bmod p$. Then the $(i, j)$ entry of $M N$ is the element of $F$ given by the sum

$$
\sum_{z \in F} m_{z} \cdot z=\sum_{z \in F} \bar{m}_{z} \cdot z
$$

Since there are only finitely many $\left(p^{q}\right)$ possible functions $z \mapsto \bar{m}_{z}$, our program or formula can contain a table giving, for each of these functions, the value of the sum.

The following proposition summarizes the preceding discussion.

Proposition 30 For any finite field $F$, there is an FP+Card definition of non-singularity for square matrices over $F$. 
At two points in the preceding discussion, we used that the field $F$ is fixed, so that our FP+Card formula can contain complete descriptions of the sets $P_{z}$ and the sums associated to the functions $z \mapsto \bar{m}_{z}$. It is not difficult, however, to adjust the algorithm to work uniformly over all finite fields $F$, in time polynomial in $|F|=q$ and the size of the matrix. In the first place, the table of all the $P_{z}$ 's is essentially the multiplication table of the field; its size is only quadratic in $q$. So this table can be computed as part of the algorithm.

There isn't enough time to compute the sums associated to all possible functions $z \mapsto \bar{m}_{z}$, since there are $p^{q}$ of these functions. But when multiplying a particular pair of matrices, we need the sum for only one such function per entry. Each single sum is easy to compute provided we are given an ordering of $F$. So there is no difficulty computing, in polynomial time, the $n^{2}$ sums actually needed. Thus, we obtain the following uniform version of the preceding proposition.

Proposition 31 There is an FP+Card formula defining non-singularity of square matrices over finite fields, where the input structure consists of a finite field $F$, a linear ordering of the set $F$, a set $I$, and an I-square matrix $M: I^{2} \rightarrow F$.

To avoid possible confusion, we point out that there is no necessary connection between the linear ordering of $F$ and the field operations.

Question 32 Can the determinant of a square matrix over a finite field be computed in $\tilde{\mathrm{CPT}}+$ Card? Can it be defined in FP+Card?

\subsection{Integer Matrices}

In this subsection, we apply the preceding results to matrices with entries from the ring $\mathbb{Z}$ of integers. Since we require inputs of computations to be finite structures for finite vocabularies, we must modify the representation of matrices as structures described in Definitions 25 and 26. Those definitions would yield an infinite vocabulary whenever the underlying ring is infinite, and if we represented the matrix by a function (as in Proposition 31) instead of a family of relations then the vocabulary would be finite but the underlying set of the structure would be infinite. We adopt the convention that matrix entries are to be written in binary notation. Recall that this means that an 
entry $r$ is represented by a set $C$ of natural numbers, the set of locations of ones in the binary expansion. Thus, each matrix entry is to be a set of natural numbers, and therefore the matrix itself amounts to a ternary relation, $M(i, j, s)$ with the meaning "the coefficient of $2^{s}$ in the binary expansion of the $(i, j)$ entry of $M$ is $1 . "$ There are two problems with this set-up.

The smaller problem is that we have not taken into account the signs of the matrix entries. So we shall need a second relation, a binary one, with the meaning "the $(i, j)$ entry of $M$ is positive."

The more serious problem is that, although the first and second arguments of the ternary relation $M$ are atoms, namely indices for rows or columns of our matrix, the third argument is a natural number. Both BGS and $\mathrm{FP}+$ Card are set up so that the numbers (von Neumann ordinals in the case of BGS, the numerical second sort in the case of FP+Card) are not part of the input structure. So $M$ is not appropriate as an input in BGS or FP+Card. We therefore include in the input structure a copy of enough of the natural number system to allow coding our binary numbers. Our official representation of integer matrices will thus involve surrogate natural numbers $\hat{0}, \hat{1}, \ldots, \hat{k}$, although for practical purposes, it does no harm to think of $0,1, \ldots, k$ instead.

That is, an $I$-square matrix $M$ will be regarded as a two-sorted structure with underlying sets $I$ and a set of indices $\{\hat{0}, \hat{1}, \ldots, \hat{k}\}$; the relations on this structure are the linear ordering $\hat{0}<\hat{1}<\cdots<\hat{k}$ on the second sort, the ternary relation $M(i, j, \hat{s})$ defined by "the coefficient of $2^{s}$ in the binary expansion of the absolute value of the $(i, j)$ entry of $M$ is 1 ," and the binary relation "the $(i, j)$ entry of $M$ is positive."

The number $k$ in this representation of a matrix $M$ would ordinarily be taken as small as possible, so it is essentially the logarithm of the largest absolute value of the matrix entries.

Theorem 33 There is an FP+Card formula which, on matrices $M$ over $\mathbb{Z}$ represented as structures as above, defines " $M$ is non-singular."

Proof We describe an algorithm for deciding whether a square matrix over $\mathbb{Z}$ is non-singular, and we show that it works, without arbitrary choices, in polynomial time. The details of formalization in $\tilde{\mathrm{CPT}}+\mathrm{Card}$ or in $\mathrm{FP}+\mathrm{Card}$ will, however, be left to the reader.

Given a square matrix $M$, represented as above by a structure with underlying sets $I$ (indexing the rows and columns) and $\{\hat{0}, \hat{1}, \ldots, \hat{r}\}$ (indexing 
the digits in each entry), the algorithm proceeds as follows. First, find the cardinalities $|I|$ and $r+1$ of these two sets, and let $n$ be the larger of the two. Thus, the size of the matrix is at most $n \times n$ and each entry is at most $2^{n}$ in absolute value. The entire algorithm will take time polynomial in $n$.

Second, generate a list of the first $2 n^{2}$ prime numbers. (For a BGS algorithm, the primes are represented as von Neumann ordinals. For an FP+Card definition, they are represented by rather short tuples of elements from the numerical sort; in fact, triples will suffice - see below.) This list can be produced by applying the sieve of Eratosthenes. The time required by the sieve of Eratosthenes is polynomial relative to the prime numbers involved (though it is not polynomial relative to the lengths of the primes in binary notation). And the primes involved here are, according to the prime number theorem, below $n^{3}$ provided $n$ is large enough. Therefore the time needed to generate this list of primes is polynomial in $n$.

Third, go through all the primes $p$ in the list, checking for each one whether $M$ reduced modulo $p$ is non-singular as a matrix over $\mathbb{Z} / p$. The results of the previous subsection show that this can be done in polynomial time.

Finally, output "yes," meaning that $M$ is non-singular, if and only if it was non-singular modulo at least one of the primes $p$ on the list.

This algorithm can clearly be programmed in BGS with the cardinality function, and it runs in polynomial time. It is a routine matter to formalize it in $\mathrm{FP}+\mathrm{Card}$. It remains to show that it gives the correct answer.

If $M$ is singular, i.e., if its determinant is zero, then its reduction modulo $p$ has determinant zero for every prime $p$. Conversely, suppose $M$ is nonsingular, and let its determinant be $d \neq 0$. Of course, then $M$ is non-singular modulo some primes, for example any primes larger than $|d|$, but we must show that it is non-singular modulo at least one of the first $2 n^{2}$ primes.

For this purpose, we first estimate how big $|d|$ might be. The determinant of a $k \times k$ matrix is the sum of $k$ ! terms, each of which is the product of $k$ of the matrix's entries. In our situation, this means that $d$ is the sum of at most $n$ ! terms, each the product of at most $n$ numbers, each at most $2^{n}$ in absolute value. Thus, each of these $n$ ! products is at most $2^{n^{2}}$. Therefore,

$$
|d| \leq n ! \cdot 2^{n^{2}} \leq n^{n} \cdot 2^{n^{2}} \leq\left(2^{n}\right)^{n} \cdot 2^{n^{2}}=2^{2 n^{2}}
$$

Recall that the list of primes used by our algorithm consisted of the first $2 n^{2}$ primes. Since each prime is $\geq 2$, the product of the listed primes is larger 
than $2^{2 n^{2}}$ and therefore larger than $|d|$. Thus, $|d|$, being non-zero, cannot be divisible by this product of primes. That means that at least one prime $p$ on our list fails to divide $|d|$, i.e., that $M$ is non-singular modulo $p$, and therefore the algorithm gives the correct answer.

Remark 34 The algorithm described in the preceding proof does more than the theorem claims. It determines exactly which primes divide the determinant $d$ of $M$. Indeed, it checks this divisibility directly for the first $2 n^{2}$ primes, and the proof shows that no larger prime can divide $d$ unless $d=0$.

The algorithm does not quite determine the value of $d$, for it does not determine its sign nor does it determine, for primes $p$ dividing $d$, whether $p^{2}$ or higher powers also divide $d$. We do not know whether the determinant of an integer matrix can be computed (as a signed binary expansion) in polynomial time by a BGS program with the cardinality function.

\subsection{Rows and Columns May Differ}

In the preceding discussion of determinants, we have dealt only with $I$-square matrices. Up to a sign, determinants make sense for $I \times J$ matrices when $|I|=|J|$, and it makes sense to ask whether non-singularity of such matrices can be computed in $\tilde{\mathrm{CPT}}+$ Card or defined in FP+Card. The algorithms from the preceding subsections do not suffice for this purpose, for they depend on taking powers of the given matrix, and $M^{2}$ is well-defined only when the rows and columns of $M$ are indexed by the same set. Nevertheless, these algorithms can be modified to work when the rows and columns are indexed by different sets of the same size.

Theorem 35 There is an FP+Card formula defining non-singularity of matrices over finite fields, where the input structure consists of a finite field $F$, a linear ordering of the set $F$, two sets $I$ and $J$ with $|I|=|J|$, and an $I \times J$ matrix $M: I \times J \rightarrow F$.

Proof Let $M$ be an $I \times J$ matrix as in the statement of the theorem. Although $M^{2}$ is not defined when $I \neq J, M \cdot M^{t}$ is defined, where the superscript $t$ means transpose. Furthermore, $M \cdot M^{t}$ is an $I$-square matrix.

Its entry in position $\left(i, i^{\prime}\right)$ is $\sum_{j \in J} M(i, j) M\left(i^{\prime}, j\right)$ which makes good sense for any $i, i^{\prime} \in I$. So by Proposition 31 we can define non-singularity of $M \cdot M^{t}$ by an $\mathrm{FP}+$ Card formula. But this is the same as defining non-singularity of 
$M$, since the determinant of $M \cdot M^{t}$ is the square of the determinant of $M$.

Remark 36 An alternative proof of the theorem uses, instead of $M \cdot M^{t}$, the block matrix

$$
\left(\begin{array}{cc}
0 & M \\
M^{t} & 0
\end{array}\right) .
$$

If $I$ and $J$ are disjoint (otherwise replace them by $I \times\{0\}$ and $J \times\{1\}$ ) then this block matrix is an $(I \cup J)$-square matrix. Its $(x, y)$ entry is 0 , if $x$ and $y$ are both in $I$ or both in $J ; M(x, y)$, if $x \in I$ and $y \in J$; and $M(y, x)$ if $x \in J$ and $y \in I$. Proposition 31 allows us to define non-singularity of this block matrix, but again this is the same as non-singularity of $M$, since the determinant of the block matrix is the square of the determinant of $M$.

\section{Open Problems}

The main problem that remains open is whether there is a logic, in the sense of [11], that captures polynomial time on unordered structures. It was conjectured in [11] that the answer is negative.

A special case of the main problem is whether $\tilde{C}$ PT+Card captures PTime. Of course a negative answer here is even more likely, but we have not been able to prove it. In view of the results in this paper, a negative answer for the special case would follow from a negative answer to any of the following questions.

- Can a C̃PT+Card program distinguish between the (unpadded) Cai, Fürer, Immerman graphs $\mathfrak{G}_{m}^{0}$ and $\mathfrak{G}_{m}^{1}$ (as defined in Section 4) for all $m$ ?

- Can isomorphism of 3-multipedes with shoes be decided by a $\tilde{\mathrm{CPT}}+$ Card program?

- Can a C̃PT+Card program decide whether a given graph (not necessarily bipartite) admits a complete matching?

- Can a $\tilde{C} P T+C a r d$ program compute, up to sign, the determinant of an $I \times J$ matrix over a finite field (where $|I|=|J|)$ ? 
We point out that, although we have formulated these questions for $\tilde{\mathrm{CPT}}+\mathrm{Card}$, the logic in which we are primarily interested, the first two of them are open also for $\tilde{\mathrm{CPT}}$, and the last two are open also for $\mathrm{FP}+\mathrm{Card}$.

\section{References}

[1] Serge Abiteboul and Victor Vianu, Generic computation and its complexity, Proc. 23rd A.C.M. Symp. on Theory of Computing (1991) 209219.

[2] ASM Michigan Web Page, http://www.eecs.umich.edu/gasm/, maintained by J. K. Huggins.

[3] Andreas Blass and Yuri Gurevich, Strong extension axioms and Shelah's zero-one law for choiceless polynomial time, to appear.

[4] Andreas Blass, Yuri Gurevich, and Saharon Shelah, Choiceless polynomial time, Ann. Pure Appl. Logic 100 (1999) 141-187.

[5] Andreas Blass, Yuri Gurevich, and Jan Van den Bussche, Abstract state machines and computationally complete query languages, abridged version in Abstract State Machines: Theory and Applications, Ed. Y. Gurevich, P. Kutter, M. Odersky, and L. Thiele, Springer-Verlag, Lecture Notes in Computer Science 1912 (2000) 22-33; full version to appear in Information and Computation.

[6] Béla Bollobás, Graph Theory: An Introductory Course, Springer-Verlag 1979.

[7] Jin-Yi Cai, Martin Fürer, and Neil Immerman, An optimal lower bound on the number of variables for graph identification, Combinatorica 12 (1992) 389-410.

[8] Ashok Chandra and David Harel, Structure and complexity of relational queries, J. Comput. System Sci. 25 (1982) 99-128.

[9] Francoise Gire and Huy Khanh Hoang, An extension of fixpoint logic with a symmetry-based choice construct, Information and Computation 144 (1998) 40-65. 
[10] Erich Grädel and Yuri Gurevich, Metafinite model theory, Information and Computation 140 (1998) 26-81.

[11] Yuri Gurevich, Logic and the challenge of computer science, in Current Trends in Theoretical Computer Science, ed. E. Börger, Computer Science Press (1988) 1-57.

[12] Yuri Gurevich, Evolving algebras 1993: Lipari guide, Specification and Validation Methods, ed. E. Börger, Oxford Univ. Press (1995) 9-36.

[13] Yuri Gurevich and Saharon Shelah, On finite rigid structures, J. Symbolic Logic 61 (1996) 549-562.

[14] Neil Immerman, Relational queries computable in polynomial time, Information and Control 68 (1986) 86-104.

[15] Martin Otto, Bounded Variable Logics and Counting: A Study in Finite Models, Springer-Verlag, Lecture Notes in Logic 9 (1997).

[16] Saharon Shelah, Choiceless polynomial time logic: inability to express [paper number 634], in Computer Science Logic 2000, editors Peter Clote and Helmut Schwichtenberg, Springer Lecture Notes in Computer Science 1862 (2000) 72-125.

[17] Moshe Vardi, The complexity of relational query languages, Proc. 14th A.C.M. Symp. on Theory of Computing (1982) 137-146. 